\title{
Nested maximin Latin hypercube designs
}

\author{
Gijs Rennen • Bart Husslage • Edwin R. Van Dam • \\ Dick Den Hertog
}

Received: 16 January 2009 / Revised: 19 August 2009 / Accepted: 22 August 2009 / Published online: 6 October 2009

(C) The Author(s) 2009. This article is published with open access at Springerlink.com

\begin{abstract}
In the field of design of computer experiments (DoCE), Latin hypercube designs are frequently used for the approximation and optimization of blackboxes. In certain situations, we need a special type of designs consisting of two separate designs, one being a subset of the other. These nested designs can be used to deal with training and test sets, models with different levels of accuracy, linking parameters, and sequential evaluations. In this paper, we construct nested maximin Latin hypercube designs for up to ten dimensions. We show that different types of grids should be considered when constructing nested designs and discuss how to determine which grid to use for a specific application. To determine nested maximin designs for dimensions higher than two, four variants of the ESE algorithm of Jin et al. (J Stat Plan Inference 134(1):268-287, 2005)
\end{abstract}

The research of B.G.M. Husslage has been financially supported by the SamenwerkingsOrgaan Brabantse Universiteiten (SOBU).

The research of E.R. Van Dam has been made possible by a fellowship of the Royal Netherlands Academy of Arts and Sciences.

\footnotetext{
G. Rennen · B. Husslage · E. R. Van Dam (凶) .

D. Den Hertog

Department of Econometrics and Operations Research, CentER, Tilburg University, P.O. Box 90153,

5000 LE Tilburg, The Netherlands

e-mail: Edwin.vanDam@uvt.nl

G. Rennen

e-mail: G.Rennen@uvt.nl

B. Husslage

e-mail: Husslage@casema.nl

D. Den Hertog

e-mail: D.denHertog@uvt.nl
}

are introduced and compared. Our main focus is on GROUPRAND, the most successful of these four variants. In the numerical comparison, we consider the calculation times, space-fillingness of the obtained designs and the performance of different grids. Maximin distances for different numbers of points are provided; the corresponding nested maximin designs can be found on the website http://www.spacefillingdesigns.nl.

Keywords Design of computer experiments • Latin hypercube design $\cdot$ Linking parameter • Multi-fidelity modeling • Nested designs •

Sequential simulation - Space-filling •

Training and test set $\cdot$ Validation

\section{Introduction}

Latin hypercube designs are very useful in the approximation of black-box functions. By definition, black-box functions have no explicit description, but can be evaluated to obtain output values for specific input values. As evaluations of a black-box function often involve time-consuming computer simulations, we would like to construct an approximating model (or metamodel) based on evaluations in a (small) number of points. See, e.g., Montgomery (1984), Sacks et al. (1989a, b), Myers (1999), Jones (2001), Booker et al. (1999), Den Hertog and Stehouwer (2002), Santner et al. (2003), Queipo et al. (2005), Wang and Shan (2007), and Kleijnen (2008). A review of metamodeling applications in structural optimization can be found in Barthelemy and Haftka (1993), and in multidisciplinary design optimization in Sobieszczanski-Sobieski and Haftka (1997) and Simpson et al. (2008). 
We will use the term design to denote the set of evaluation points. As observed by many researchers, there is an important distinction between designs for computer experiments and designs of experiments for the more traditional response surface methods. Physical experiments exhibit random errors whereas computer experiments are often deterministic (see e.g. Simpson et al. 2004; Forrester et al. 2006, 2008). Therefore, designs for experiments often evaluate certain points multiple times. For designs for computer experiments, replication is redundant because the same input will always result in the same output. This distinction is crucial, so one of the main aims in the field of design of computer experiments (DoCE) is therefore to obtain efficient designs for computer experiments.

As is recognized by several authors, a design for computer experiments should at least satisfy the following two criteria (see Johnson et al. 1990 and Morris and Mitchell 1995). First of all, the design should be spacefilling in some sense. When no details on the functional behavior of the response parameters are available, it is important to be able to obtain information from the entire design space. Therefore, design points should be "evenly spread" over the entire region. Secondly, the design should be non-collapsing. When one of the design parameters has (almost) no influence on the black-box function value, two design points that differ only in this parameter will "collapse", i.e., they can be considered as the same point that is evaluated twice. As evaluation of the deterministic black-box function is often time-consuming, this is not a desirable situation. Therefore, two design points should not share any coordinate values when it is not known a priori which parameters are important. Moreover, we would like the projections of the points onto the axes to be separated as much as possible. When we consider a black-box function on a box-constrained domain, this can be accomplished by using Latin hypercube designs. A Latin hypercube design (LHD) of $n$ points in $m$ dimensions can be defined as an $n \times m$ matrix, were each column is a permutation of the set $\left\{0, \frac{1}{n-1}, \frac{2}{n-1}, \ldots, 1\right\}$. The rows $x_{i}=\left(x_{i 1}, x_{i 2}, \ldots, x_{i m}\right), i=1, \ldots, n$, then define the $n$ design points. Because the columns are permutations of the above set, for all of the $m$ coordinates it holds that no two design points have the same value.

To obtain space-filling designs, the evaluation points are chosen in such a way that the separation distance (i.e., the minimal distance among any pair of points) is maximized, leading to so-called maximin designs. Other space-filling designs, like minimax, integrated mean squared error (IMSE), Audze-Eglais, discrepancy and maximum entropy designs, are also used in the literature. For a good survey of these designs see the book of Santner et al. (2003). Goel et al. (2008) argue that it would be better to use several criteria when selecting a design. However, Santner et al. (2003) show that that maximin Latin hypercube designs generally speaking yield good approximations.

Maximin Latin hypercube designs were first constructed by Morris and Mitchell (1995) using simulated annealing. Ye et al. (2000) consider only the class of symmetric approximate maximin LHDs to reduce the computing effort. Jin et al. (2005) introduce the enhanced stochastic evolutionary (ESE) algorithm for finding various space-filling designs, including approximate maximin LHDs. Husslage et al. (2008) use the ESE algorithm to construct approximate maximin LHDs for up to 10 dimensions and up to 300 design points. Furthermore, they also construct approximate maximin LHDs by optimizing the maximin criterion over all LHDs having a certain periodic structure. This approach is an extension of the method used in Van Dam et al. (2007) to obtain two-dimensional approximate maximin LHDs. In that paper, two-dimensional maximin LHDs are also found using a branch-andbound algorithm. Finally, Grosso et al. (2009) use Iterated Local Search heuristics to find good approximate maximin LHDs for up to 10 dimensions. The best designs found in these papers are published on-line at http://www.spacefillingdesigns.nl. This website also contains the upper bounds on the separation distance for certain classes of maximin LHDs found by Van Dam et al. (2009b). These upper bounds can be used to asses the quality of approximate maximin LHDs.

In real-life, there are situations where we need a special type of designs called nested designs. This type of design consists of two separate designs, with the requirement that one design is a subset of the other design. Van Dam et al. (2009a) show how to construct one-dimensional nested maximin designs; the current paper focuses on two and higher dimensional designs. ${ }^{1}$ Four main reasons for nesting maximin designs are: validation, models with different levels of accuracy, linking parameters, and sequential evaluations.

To start with the first reason, consider the problem of fitting and validating a particular metamodel. In practice, the following approach is often used. First, a metamodel is fitted to the responses obtained when evaluating the design points in the training set. Then, a new set of design points, i.e., the test set, is evaluated and the obtained responses are compared to the response values predicted by the metamodel. If the differences between the predicted and the actual

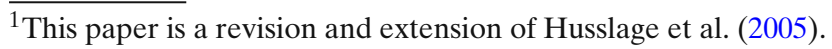


response values are small, the metamodel is considered to be valid. See also Cherkassky and Mulier (1998) for a more detailed description of the use of training and test sets. Because a metamodel should be a global approximation model, i.e., it should be valid for the entire feasible region, both the training set and the test set should cover the entire region. Moreover, the design points in the test set should not lie too close to the design points in the training set, i.e., the total set of design points should be space-filling. This can be accomplished by nesting two designs, which are optimized with respect to, for example, the maximin criterion. The design points that are in both designs then form the training set and the points that are only in the large design make up the test set.

The second motivation comes from the situation where an output variable of a process, product, or system is modeled by two black-box functions with different levels of accuracy. These black-box functions could, for instance, be simulation models with different levels of detail. As a more accurate model is in general also more time-consuming, we can perform fewer evaluations of the high accuracy model than of the low accuracy model in the same amount of time. Instead of choosing to use either the high or low accuracy model, we can also choose to use both. We can evaluate the high accuracy model at all points in the small design and the low accuracy model at all points in the large design. By using a nested design, high and low accuracy evaluations are thus performed at all points in the small design. Multi-fidelity methods can combine the results from both models to obtain a metamodel that is better than a metamodel obtained by using only one of the two models and the same amount of time. More information on multi-fidelity methods can be found in Cressie (1993), Kennedy and O'Hagan (2000), Qian et al. (2006), and Forrester et al. (2007).

Another reason for using nested designs is caused by linking parameters. Consider a product that consists of two components, each of them represented by a separate black-box function. To obtain an approximating model describing the behavior of the complete product, function evaluations of each black-box function are needed. When one black-box function is more timeconsuming to evaluate than the other, it could be better to perform different numbers of function evaluations of each black-box function. Moreover, in practice it may occur that these functions have input parameters in common; such parameters are called linking parameters, see Husslage et al. (2003). Evaluating the linking parameters at the same setting in both functions (i.e., component-wise) leads to an evaluation of the product. Not only do product evaluations provide a better un- derstanding of the product, they are also very useful in the product optimization process. Another reason for using the same settings for (linking) parameters is due to physical restrictions on the black-box functions. Setting the parameters for computer experiments can be a time-consuming job in practice, because characteristics, like shape and structure, have to be redefined for every new experiment. Therefore, it is preferable to use the same settings as much as possible. By constructing nested designs we can determine the settings for linking parameters.

Nested designs are also useful when dealing with sequential evaluations. In practice it is common that after evaluating an initial set of points, extra evaluations are needed. As an example, suppose we construct an approximating model for some black-box function based on $n_{1}$ function evaluations. However, after validating the obtained model it turns out that an extra set of function evaluations is needed to build a proper model. We then face the problem of constructing a design on a total of, say, $n_{2}$ points, given the initial design on $n_{1}$ points. To anticipate the possibility of extra evaluations, one can construct the two designs (on $n_{1}$ and $n_{2}$ points) at once, hence, by constructing a nested design. An alternative method to deal with this situation would be sequential sampling. As this is beyond the scope of this paper, we refer to Jones (2001) and Jin et al. (2002) for more information on sequential sampling.

We have just described why both Latin hypercube designs and nested designs are important. In this paper we construct nested maximin Latin hypercube designs in $m$ dimensions with $m \geq 2$. Section 2 gives a more detailed formulation of this problem. When nesting two designs, it is not always possible to satisfy the LHDstructure for both designs. Therefore, we introduce in Section 3 three different grid-structures which approximate the LHD-structure as good as possible. Furthermore, we discuss how to select a suitable grid-structure for a particular application. In Section 4, a branchand-bound method for determining two-dimensional nested maximin designs is presented and Pareto optimal nested designs in two dimensions are discussed. For higher dimensions, determining nested maximin designs, i.e. nested designs which maximize the scaled separation distance $d$, becomes too time consuming. Therefore in Section 5, we introduce a heuristic which also aims to maximize $d$ but does not guarantee to find the optimal $d$. We refer to the resulting designs as nested approximate maximin designs. In Section 6, numerical results obtained with this heuristic and the branch-and-bound method are presented and discussed. Finally, Section 7 contains concluding remarks. 


\section{Problem formulation}

In this paper, we focus on the problem of nesting two designs, $X_{1}$ and $X_{2}$, with $X_{1} \subset X_{2}, X_{j}=\left\{x_{i}=\right.$ $\left.\left(x_{i 1}, x_{i 2}, \ldots, x_{i m}\right) \mid i \in I_{j}\right\}$, and $\left|I_{j}\right|=n_{j}, j=1,2$. Thus, the index set $I_{1} \subset I_{2}=\left\{1,2, \ldots, n_{2}\right\}$ defines which design points $x_{i}$ are part of both designs. The nested design is defined by the combination of $X_{1}$ and $X_{2}$. All design parameters are scaled such that they take values in the interval $[0,1]$.

The first condition which we impose on the nested designs is that $X_{1}$ and $X_{2}$ must both be non-collapsing. This can be accomplished by using the LHD-structure. The main property of a regular LHD is that all points, when projected onto one of the axes, are equidistantly distributed. To form an LHD, the points in $X_{1}$ must thus be projected onto the set $\left\{0, \frac{1}{n_{1}-1}, \frac{2}{n_{1}-1}, \ldots, 1\right\}$ and the points in $X_{2}$ onto $\left\{0, \frac{1}{n_{2}-1}, \frac{2}{n_{2}-1}, \ldots, 1\right\}$. In order for $X_{1}$ and $X_{2}$ to both form a Latin hypercube design, the first set must be a subset of the second. However, this only holds when $n_{2}-1$ is a multiple of $n_{1}-1$ or, stated differently, when

$c_{2}:=\frac{n_{2}-1}{n_{1}-1}$

is integer. In all other cases, we have to compromise on the LHD-structure of one or both designs. As there are different ways of doing this, we propose three different grid-structures, nested $n_{1}$-grids, nested $n_{2}$-grids and grids with nested maximin axes, in Section 3. All of these grid-structures are constructed to compromise as little as possible on the LHD-structure. When $c_{2}$ is integer, the different grid-structures coincide and are such that both $X_{1}$ and $X_{2}$ are LHDs.

Secondly, we aim to determine the design points $x_{i}$ and the set $I_{1}$ such that both designs are as much as possible space-filling given the chosen grid-structure. To optimize the space-fillingness, we choose to use the maximin distance criterion. As the distances between the points in $X_{1}$ will naturally be greater than the distances between the points in $X_{2}$, scaling of these distances is necessary to enable a fair comparison with the maximin distance criterion. Therefore, we define scaled separation distance $d_{j}$ as the minimal scaled distance between all points in the design $X_{j}$ :

$d_{j}:=\min _{\substack{k, l \in I_{j} \\ k \neq l}} \frac{d\left(x_{k}, x_{l}\right)}{s_{j}}, j=1,2$,

where $d(\cdot, \cdot)$ is the Euclidean distance measure and $s_{1}$ and $s_{2}$ are scaling factors for the Euclidean distances in $X_{1}$ and $X_{2}$, respectively. Because one-dimensional designs of $n$ points have distance $1 /(n-1)$ and the minimum distance of $n$ points in an $m$-dimensional hypercube is at most of the order $1 / \sqrt[m]{n-1}$, it seems natural to use scaling factors

$s_{j}:=1 / \sqrt[m]{n_{j}-1}, \quad j=1,2$,

in (1) for $m$-dimensional designs. As we use the maximin distance criterion, we have to maximize the minimal scaled distance between any pair of points in $X_{1}$ and $X_{2}$. Therefore, what remains is to maximize the minimal separation distance

$d=\min \left\{d_{1}, d_{2}\right\}$

over all $I_{1} \subset I_{2}$, with $\left|I_{1}\right|=n_{1}$, and $x_{i} \in[0,1]^{m}$.

We are aware that the above formulation is just one way of combining the two scaled separation distances into one objective and that other scaling factors or formulations are also possible. Using different scaling factors is no problem as all methods discussed in this paper can also be used for other values of $s_{1}$ and $s_{2}$. In Section 7, we will discuss some other alternative objectives. Dealing with maximizing $d_{1}$ and $d_{2}$ as a biobjective optimization problem is another possibility. For two-dimensional nested designs with small $n_{1}$ and $n_{2}$, we use this approach in Section 4.2. However, to limit the scope of this paper, our main focus will be on the above maximin objective.

By limiting the choice of design points to certain grids to obtain non-collapsingness, we generally obtain less space-filling designs. However, as a comparison of two-dimensional non-nested designs in Van Dam et al. (2007) shows, the loss in space-fillingness by imposing the LHD-structure is quite small. Furthermore, the non-collapsingness achieved by the LHD-structure is important when dealing with deterministic computer experiments. Especially when black-box function evaluations are expensive, using, for instance, a separate screening design to determine the significant design parameters is often not an option. Consequently, we reckon that the benefit of non-collapsingness justifies a limited loss in space-fillingness. By determining spacefilling nested LHDs, we aim to limit this loss as much as possible.

\section{Grid-structures for nested Latin hypercube designs}

As mentioned in the previous section, $X_{1}$ and $X_{2}$ can only both form a Latin hypercube design if $c_{2}:=\frac{n_{2}-1}{n_{1}-1}$ is integer. When $n_{1}$ and $n_{2}$ do not satisfy this condition, we have to use a different structure which compromises on the LHD-structure of one or both designs. In this section, we introduce three different grid-structures 


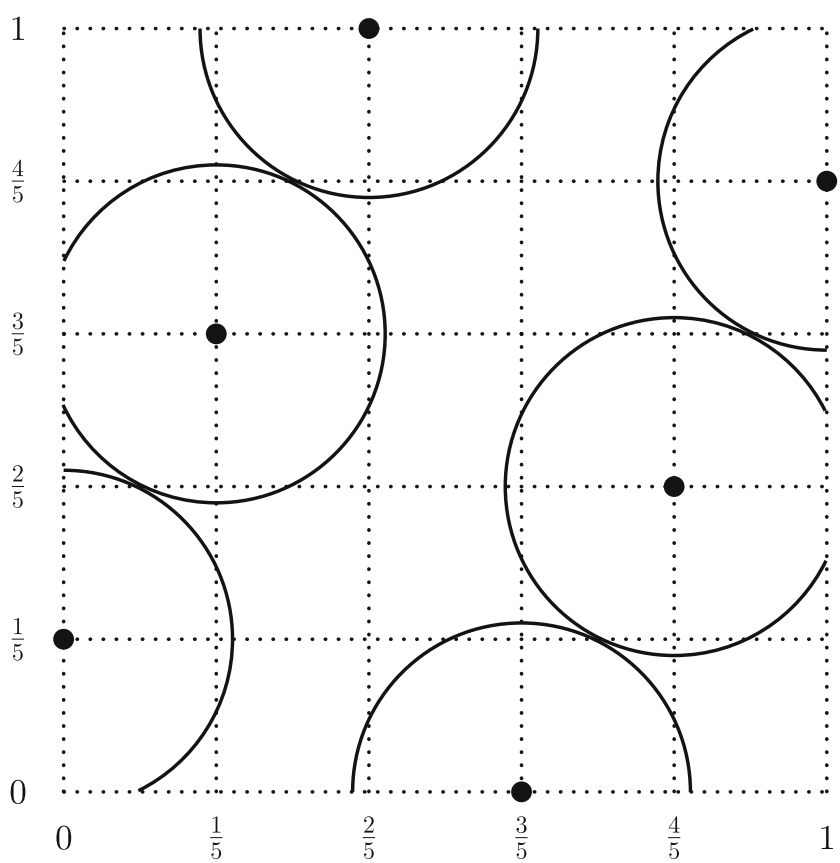

Fig. 1 A maximin Latin hypercube design of 6 points; $d_{1}=$ 1.0000

which represent different compromises. Furthermore, we discuss how to decide which grid-structure is most suitable for a particular situation.

To illustrate the different structures, examples are provided for the two-dimensional case of $n_{1}=6$ and $n_{2}=13$ points. In Figs. 1 and 2 also the individual maximin Latin hypercube designs of $n_{1}=6$ and $n_{2}=13$ points are depicted to enable comparison with the nonnested case. To compare the design in Fig. 1 with $X_{1}$ designs and the design in Fig. 2 with $X_{2}$ designs, we calculated the values of $d_{1}$ and $d_{2}$ for these non-nested designs. The circles illustrate the unscaled separation distance because when we draw circles with the design points as their centers, the separation distance is equal to the largest diameter such that the circles are nonoverlapping. Moreover, it shows where the separation distance is attained.

\subsection{Nested $n_{2}$-grid}

Before we explain the nested $n_{2}$-grid, let us first introduce the term $X_{j}$-coordinates. With $X_{j}$-coordinates, we denote the levels obtained when projecting the design points of design $X_{j}$ onto one of the axes (or dimensions), for $j=1,2$. For $X_{j}$ to be an LHD, the $X_{j}$-coordinates must thus be equidistantly distributed for every dimension.

To construct a nested design where $X_{2}$ is an LHD, we have to choose all design points on the $n_{2}$-grid, with grid points $\left\{0, \frac{1}{n_{2}-1}, \frac{2}{n_{2}-1}, \ldots, 1\right\}^{m}$. Remember that

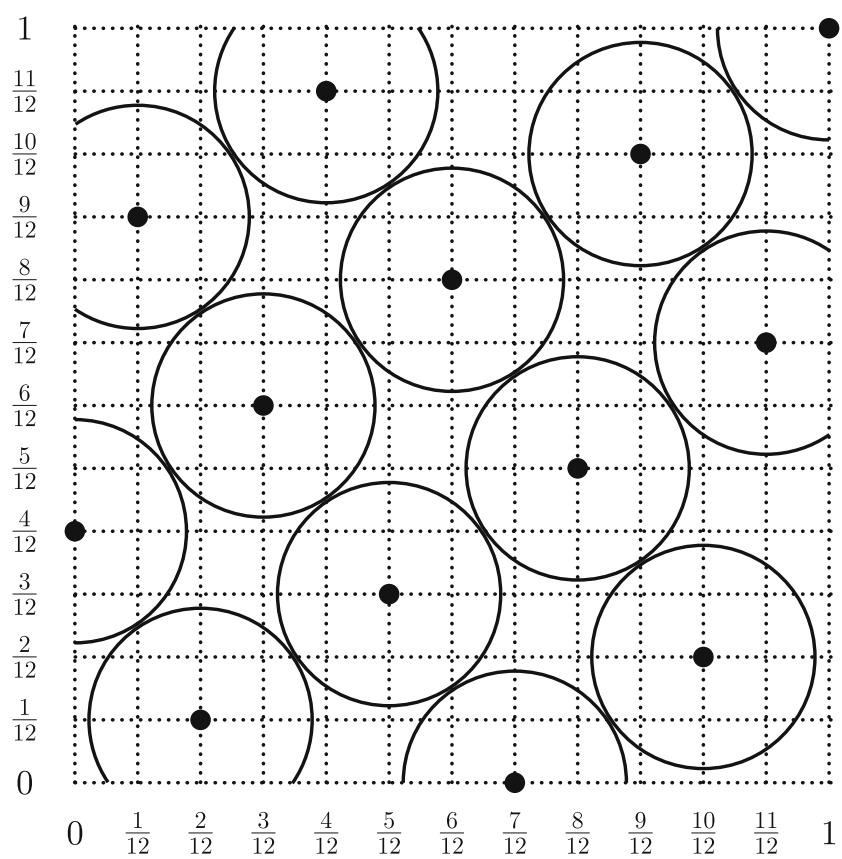

Fig. 2 A maximin Latin hypercube design of 13 points; $d_{2}=$ 1.0408

we selected the LHD-structure because of the noncollapsingness with respect to the projections of the design points onto the axes. For the design $X_{2}$, the non-collapsingness is guaranteed by the equidistant distribution of the $X_{2}$-coordinates. To obtain a noncollapsing design $X_{1}$, we also want to select the $X_{1}$ coordinates equidistantly distributed. If this is not possible, we try to obtain a space-filling distribution of the $X_{1}$-coordinates. Hence, what remains is to add restrictions that lead to the desired distribution of the $X_{1}$-coordinates.

To start, consider the case where $c_{2}=\frac{n_{2}-1}{n_{1}-1} \in \mathbb{N}$. In this case, a non-collapsing design $X_{1}$ is obtained by limiting the choice of design points (of $X_{1}$ ) to the set of equidistantly distributed $X_{1}$-coordinates $\left\{0, \frac{1}{n_{1}-1}, \frac{2}{n_{1}-1}, \ldots, 1\right\}^{m}$. See, for example, the twodimensional nested maximin Latin hypercube design of $n_{1}=16$ and $n_{2}=31$ points (with $c_{2}=2$ ) depicted in Fig. 3. As all grid-structures coincide when $c_{2}$ is integer, this design is also a nested maximin design for the other two grid-structures. Therefore, we refer to it as a nested maximin LHD instead of a nested maximin $n_{2}$-LHD.

For the case $c_{2} \notin \mathbb{N}$, the situation is more complicated. Because we are bound to the $n_{2}$-grid, and $n_{1}-1$ is no longer a divisor of $n_{2}-1$, it is no longer possible to have the $X_{1}$-coordinates equidistantly distributed. From the one-dimensional case, however, we know that for equidistantly distributed $X_{2}$-coordinates (as is the case with the $n_{2}$-grid) it is optimal to have either $\left\lfloor c_{2}\right\rfloor-$ 1 or $\left\lceil c_{2}\right\rceil-1 \quad X_{2}$-coordinates between succeeding 


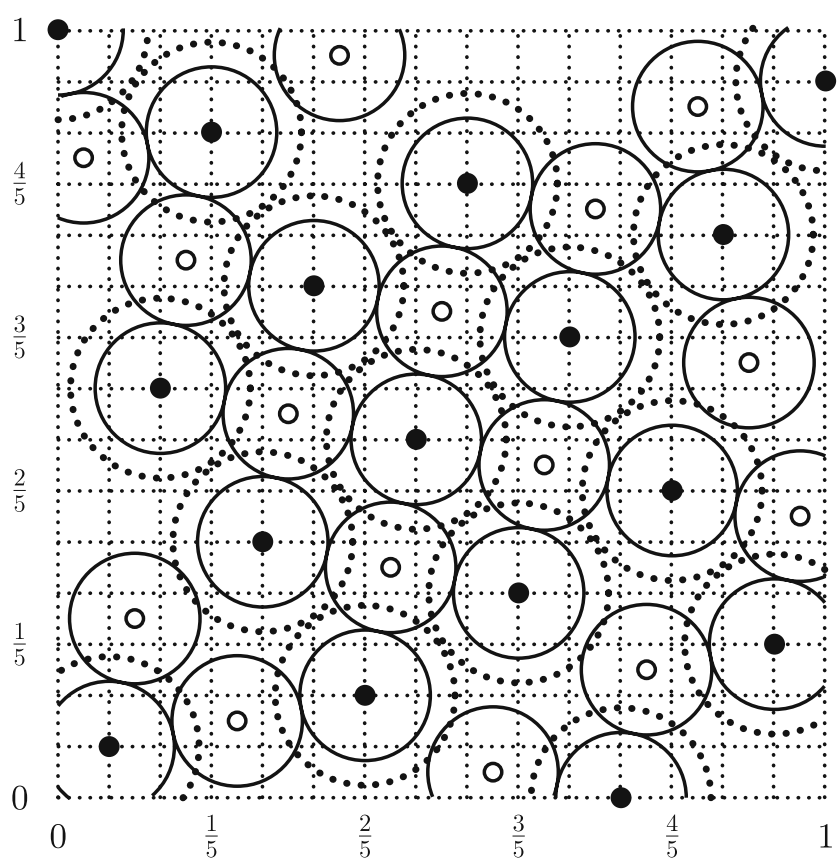

Fig. 3 A nested maximin Latin hypercube design of $n_{1}=16$ and $n_{2}=31$ points; $d=d_{1}=d_{2}=0.9309$

$X_{1}$-coordinates; see Van Dam et al. (2009a) (where $\lfloor\cdot\rfloor$ and $\lceil\cdot\rceil$ represent the floor and ceiling function, respectively). Therefore, the $X_{1}$-coordinates are required to be separated by either $\left\lfloor c_{2}\right\rfloor \frac{1}{n_{2}-1}$ or $\left\lceil c_{2}\right\rceil \frac{1}{n_{2}-1}$. Hence, should the design then collapse (onto one of the axes), then it collapses onto an optimal one-dimensional nested maximin design.

Note that this restriction still leaves multiple grids possible for design $X_{1}$ when $c_{2} \notin \mathbb{N}$. An example of a nested maximin design on a nested $n_{2}$-grid of $n_{1}=$ 6 and $n_{2}=13$ points, with $d=d_{2}=0.9129$ and $d_{1}=$ 1.0035, is depicted in Fig. 4. In this and following figures, the design points of $X_{1}$ are represented by solid dots, the open dots represent the extra design points needed to complete design $X_{2}$, hence, the solid and open dots together form the design points of $X_{2}$. The diameters of the dotted and solid circles are equal to the unscaled distance $d_{1} * s_{1}$ and $d_{2} * s_{2}$, respectively. They thus illustrate the separation distances of the designs $X_{1}$ and $X_{2}$.

For the nested $n_{2}$-grid, a suitable method to determine nested LHDs would seem to take an existing LHD of $n_{2}$-points for $X_{2}$ and select a subset of $n_{1}$ points for $X_{1}$. Forrester et al. (2007), for instance, use an exchange algorithm to implement this approach for multi-fidelity modeling. Although this method is quite attractive because of its simplicity, it does not generally yield a nested LHD satisfying all the restrictions of the nested $n_{2}$-grid. We will illustrate this with the example in Fig. 5. The figure shows a maximin Latin hypercube design for $n=15$ obtained in Van Dam

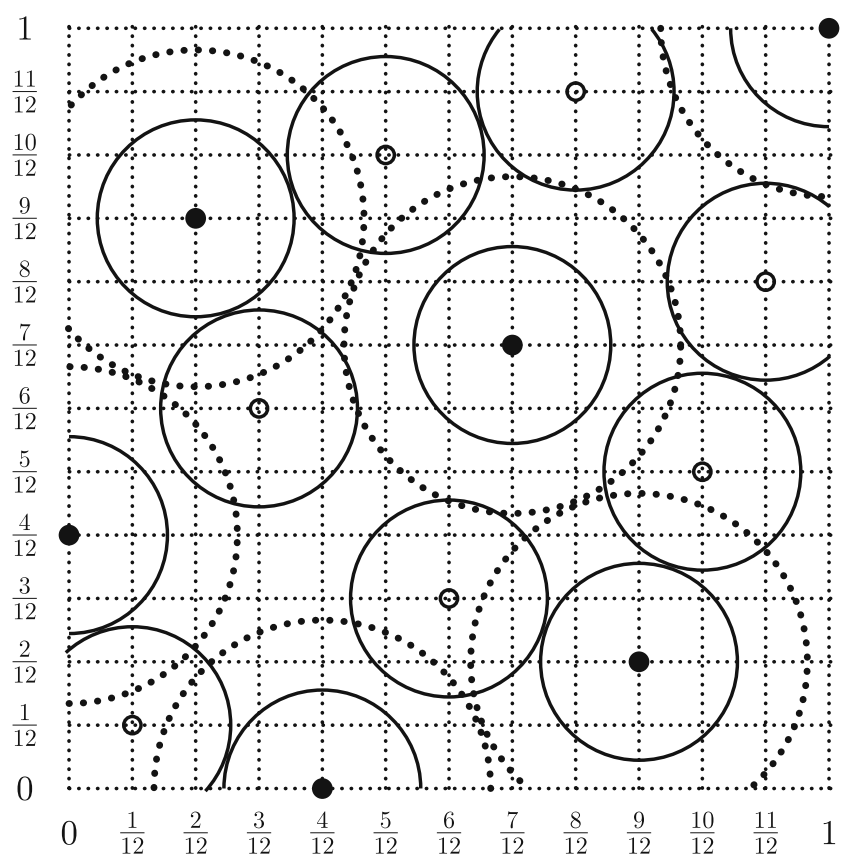

Fig. 4 A nested maximin $n_{2}$-Latin hypercube design of $n_{1}=6$ and $n_{2}=13$ points; $d=d_{2}=0.9129$ and $d_{1}=1.0035$

et al. (2007). Assume we want to construct a nested LHD with $n_{1}=8$ and $n_{2}=15$. Because in this case $c_{2}=2$, the nested $n_{2}$-grid is unique and both the $X_{1}$ and $X_{2}$-coordinates must be equidistantly distributed for both dimensions. The solid dots represent $X_{1}$ when we satisfy this latter restriction for the dimension on the horizontal axis. We can easily see that the distribution

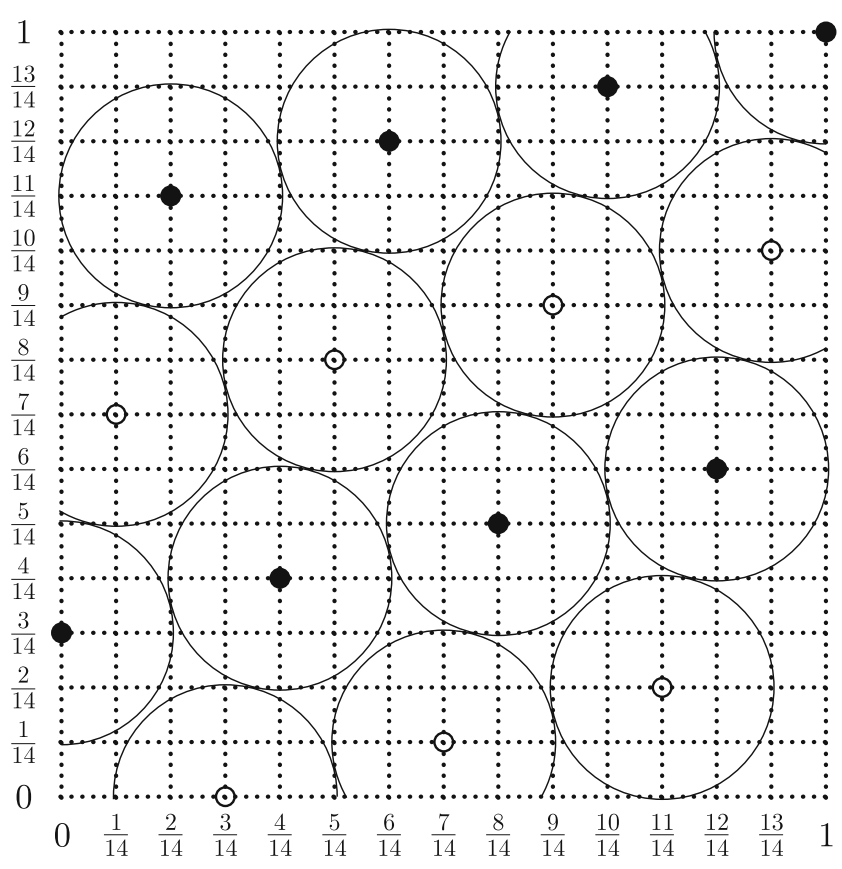

Fig. 5 Example of the problem occurring when taking $X_{1}$ equal to a subset of an existing LHD 
of the $X_{1}$-coordinates on the other axis is certainly not equidistant or space-filling. This problem also occurs for many other Latin hypercube designs and is even more likely to occur when the number of dimensions increases. Therefore, we will not use this method to construct nested LHDs, but use the methods described in Sections 4.1 and 5.1.

\subsection{Nested $n_{1}$-grid}

When we want $X_{1}$ to be an LHD instead of $X_{2}$, we can use the nested $n_{1}$-grid. The design $X_{1}$ is then obtained by choosing $n_{1}$ design points on the $n_{1}$-grid, with grid points $\left\{0, \frac{1}{n_{1}-1}, \frac{2}{n_{1}-1}, \ldots, 1\right\}^{m}$. The additional $X_{2}$ coordinates are placed equidistantly between the $X_{1}$ coordinates. Similar to the nested $n_{2}$-grid, the (interiors of the) intervals formed by consecutive $X_{1}$-coordinates are again required to contain either $\left\lfloor c_{2}\right\rfloor-1$ or $\left\lceil c_{2}\right\rceil-1$ $X_{2}$-coordinates. Hence, consecutive $X_{2}$-coordinates will be separated by either $\frac{1}{\left\lfloor c_{2}\right\rfloor} \frac{1}{n_{1}-1}$ or $\frac{1}{\left\lceil c_{2}\right\rceil} \frac{1}{n_{1}-1}$. Again, this leaves multiple grids possible when $c_{2} \notin \mathbb{N}$. See Fig. 6 for an example of a nested maximin design on a nested $n_{1}$-grid of $n_{1}=6$ and $n_{2}=13$ points, with $d=d_{2}=0.9522$ and $d_{1}=1.0000$.

\subsection{Grid with nested maximin axes}

The use of the Latin hypercube structure in the construction of a nested maximin design implies a prefer-

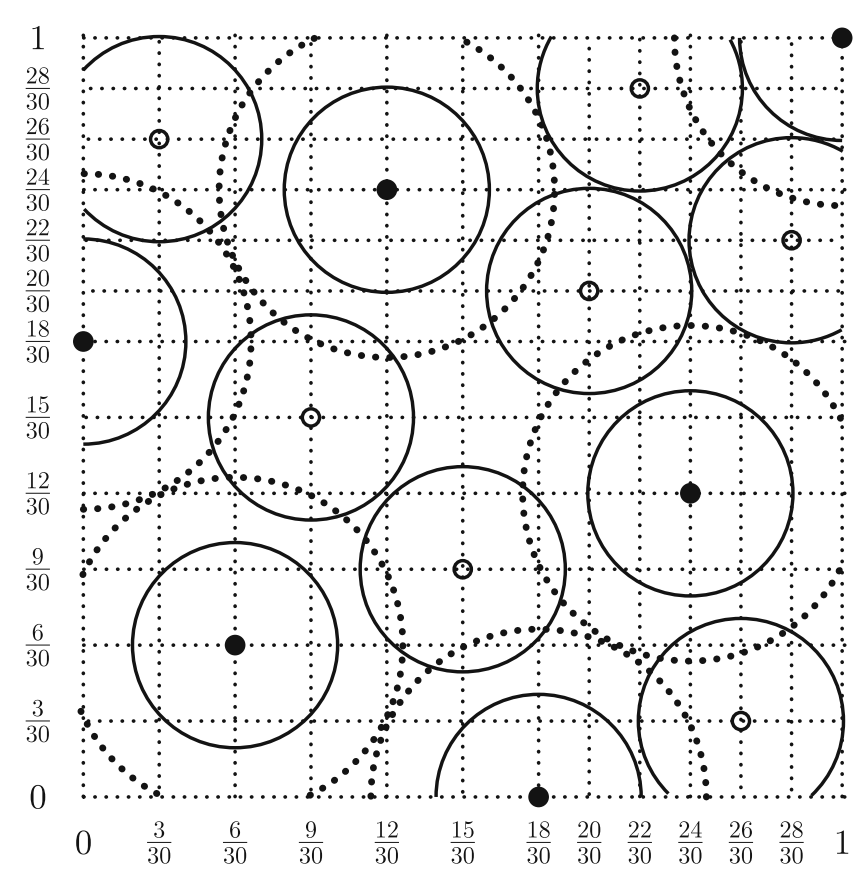

Fig. 6 A nested maximin $n_{1}$-Latin hypercube design of $n_{1}=6$ and $n_{2}=13$ points; $d=d_{2}=0.9522$ and $d_{1}=1.0000$ ence of one design over the other. Design $X_{1}$ is assumed to be more important than design $X_{2}$ when a nested $n_{1}$ grid is used; design $X_{2}$ is preferred over design $X_{1}$ in case of a nested $n_{2}$-grid. If both sets are assumed to be of equal importance, we would like to treat them equally. To deal with this problem, the $X_{1}$ - and $X_{2}$ coordinates could be restricted to take only values at the levels of a (known) one-dimensional nested maximin design of $n_{1}$ and $n_{2}$ points; see Van Dam et al. (2009a). The design points of $X_{1}$ and $X_{2}$ could then be chosen from the grid points obtained in this way. Note that in this case the projections of the design points onto the axes are always optimally space-filling with respect to the maximin distance criterion. Furthermore, note that a one-dimensional maximin design, with $c_{2} \notin \mathbb{N}$, is (again) not unique, so there are multiple grids possible. Figure 7 depicts an example of a nested maximin design of $n_{1}=6$ and $n_{2}=13$ points on a grid with nested maximin axes, with $d=d_{1}=0.9589$ and $d_{2}=0.9805$.

\subsection{Choice of grid-structure}

When deciding which grid-structure to use, there are a number of factors which can influence this decision. Firstly, when the space-fillingness of the nested design is very important, we can base our choice on which gridstructure results in the nested design with the highest $d$. Secondly, when it is not known a priori which design parameters are significant, the non-collapsingness

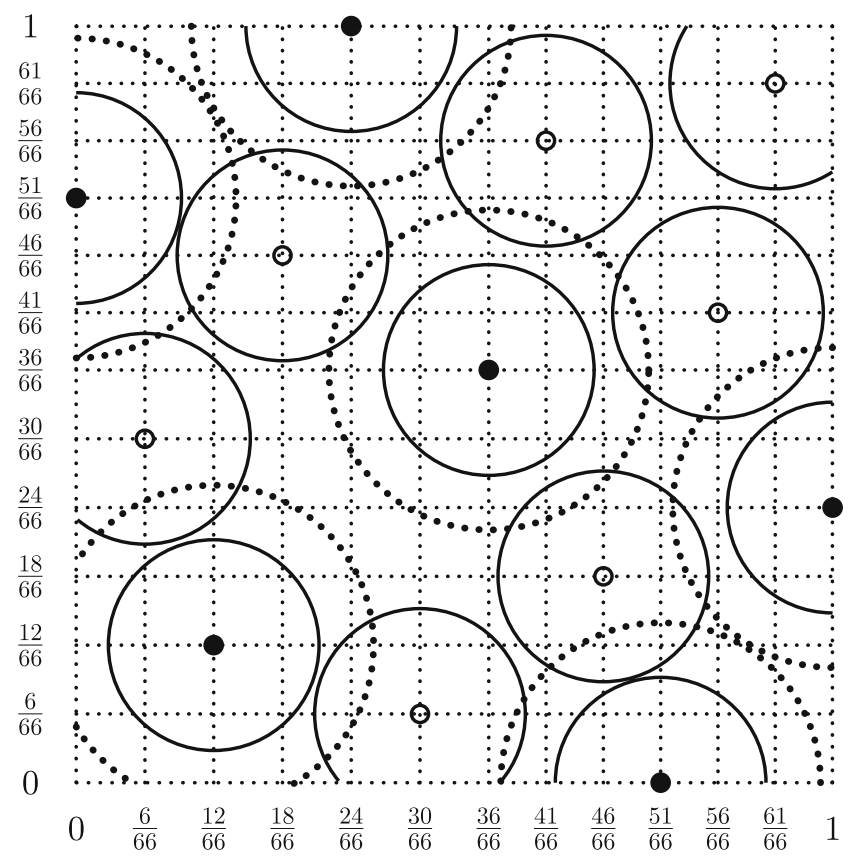

Fig. 7 A nested maximin design of $n_{1}=6$ and $n_{2}=13$ points on a grid with nested maximin axes; $d=d_{1}=0.9589$ and $d_{2}=0.9805$ 
criterion should be considered. The projections of the design point onto the axes should preferably be spacefilling, which is accomplished by choosing a grid with nested maximin axes.

Furthermore, the reason why a nested design is used may also affect the choice for a particular grid. For example, a nested $n_{1}$-grid or a grid with the highest $d_{1}$ could be preferable for sequential evaluations, because it is known with certainty that the first set of design points is evaluated, whereas the evaluation of an extra set of design points may depend on the previously evaluated set. However in the same setting, a nested $n_{2}$-grid is preferable when the final set of design points (i.e., $X_{2}$ ) is required to be a Latin hypercube design, as is often the case in practice. When dealing with linking design parameters, the choice for a specific grid mostly depends on the question which of the two designs, i.e., $X_{1}$ or $X_{2}$, is considered to be the most important one and should, thus, have an LHD-structure or have the largest separation distance. A grid with nested maximin axes should be used when there is no explicit preference for either one of the designs. When constructing a training set and a test set, design $X_{1}$, which forms the training set, is in general the most important of the two designs. This is because the prediction accuracy of a metamodel is, among others, affected by the choice of the evaluation points in the training set. A space-filling distribution of these points over the feasible region is desirable and, hence, the grid for which the design points of $X_{1}$ have the largest separation distance may be preferred. When combining high and low accuracy models, it is hard to say which of the two designs is more important. The $X_{2}$ design is important because it is used to fit the initial model, but the $X_{1}$ design is also important as it is used to evaluate the accurate model whose results must improve the initial model.

From this discussion it follows that the notion of the "best" grid-structure depends on the application under consideration and the user's preferences. Fortunately, when $c_{2} \in \mathbb{N}$, the comparison of the various nested griddesigns is superfluous. In this case, we do not have to differentiate between different grid-structures, because they will all yield the same nested maximin design (and maximin distance).

\section{Two-dimensional nested designs}

\subsection{Branch-and-bound algorithm}

To obtain two-dimensional nested maximin LHDs, we use an extension of the branch-and-bound algorithm of Van Dam et al. (2007). This extended branch-and- bound algorithm works as follows. Given $n_{1}, n_{2}$ and the grid-structure, we first determine all possible nested grids and calculate the possible distances that can occur for $X_{1}$ and $X_{2}$. These distances form a discrete set that can be efficiently searched and optimized. To determine whether a nested LHD exists with $X_{1}$ and $X_{2}$ having scaled separation distances at least $d_{1}$ and $d_{2}$, respectively, a branch-and-bound search is performed for each possible nested grid. This branch-and-bound method is similar to the one used for usual LHDs as described in Van Dam et al. (2007), however in general the nested grid-structures do not allow for the refinements given there. In the search tree, a node at level $t$ corresponds to a partial nested design consisting of $t$ design points $\left(x_{1}, \ldots, x_{t}\right)$, where the first $n_{1}$ points are in $X_{1}$ and the points are, furthermore, ordered by increasing first coordinate. Nodes in the tree are pruned when they correspond to partial nested designs that are collapsing or that have scaled separation distances smaller than $d_{1}$ or $d_{2}$.

Using the extended branch-and-bound algorithm, we obtained results for $n_{2}$ up to 15 for all three gridstructures. For the cases where $c_{2} \in \mathbb{N}$, the algorithm is refined so that nested maximin LHDs could be obtained for $n_{2}$ up 32. The maximin distances of these designs can be found in Tables 9 and 10 in Appendix B. The corresponding designs can be found on the website http://www.spacefillingdesigns.nl. In Section 6, the results are compared and discussed.

\subsection{Pareto nested designs}

Besides nested designs that maximize the objective function $d=\min \left\{d_{1}, d_{2}\right\}$, there are also some other interesting nested designs to consider: Pareto nested designs. We will call a combination of distances $\left(d_{1}, d_{2}\right)$ Pareto optimal (or Pareto) if it is not possible to improve one of the distances, without deteriorating the other distance. A Pareto nested design is a nested design of which the distances $\left(d_{1}, d_{2}\right)$ form a Pareto combination. For $c_{2} \in \mathbb{N}$ and $n_{2} \leq 32$, we have found all the Pareto combinations using a slightly adjusted version of the branch-and-bound algorithm. Furthermore, the original branch-and-bound algorithm already ensures that the distances $\left(d_{1}, d_{2}\right)$ of all nested maximin designs provided in Table 9 (see Appendix B) are Pareto optimal.

Table 1 provides all Pareto combinations $\left(d_{1}, d_{2}\right)$ corresponding to the pairs $\left(n_{1}, n_{2}\right)$, with $c_{2} \in \mathbb{N}$ and $n_{2} \leq 32$, for which there exist more than one such combination. In this table, the first entry corresponds to the optimal maximin combination $\left(d_{1}, d_{2}\right)$, followed by the other Pareto combination(s). Note that in case 
Table 1 All two-dimensional pairs $\left(n_{1}, n_{2}\right)$ with more than one Pareto combination; $c_{2} \in \mathbb{N}, n_{2} \leq 32$

\begin{tabular}{lll}
\hline$n_{1}$ & $n_{2}$ & Pareto combinations $\left(d_{1}, d_{2}\right)$ \\
\hline 4 & 10 & $(0.8165,0.9428),(1.2910,0.7454)$ \\
4 & 16 & $(1.2910,0.9309),(0.8165,1.0646)$ \\
6 & 16 & $(1.0000,0.7303),(0.6325,1.0646)$ \\
9 & 17 & $(1.1180,0.7906),(0.7906,1.0607)$ \\
4 & 19 & $(1.2910,0.9718),(0.8165,1.0000)$ \\
7 & 19 & $(1.1547,0.9718),(0.5774,1.0000)$ \\
10 & 19 & $(1.0541,0.7454),(0.7454,1.0000)$ \\
11 & 21 & $(1.0000,0.7071),(0.7071,1.0000)$ \\
8 & 22 & $(1.0690,0.8997),(0.5345,0.9258)$ \\
12 & 23 & $(0.8528,1.0871),(0.9535,0.6742)$ \\
4 & 25 & $(1.2910,1.0206),(0.8165,1.0408)$ \\
5 & 25 & $(1.1180,0.9129),(0.7071,1.0408)$ \\
7 & 25 & $(1.1547,0.8660),(0.5774,1.0408)$ \\
9 & 25 & $(1.0000,1.0408),(1.1180,0.9129)$ \\
14 & 27 & $(1.0000,1.0000),(1.1435,0.8321)$ \\
4 & 28 & $(1.2910,0.9623),(0.8165,0.9813)$ \\
10 & 28 & $(0.9428,0.9813),(1.0541,0.8607)$ \\
5 & 29 & $(1.1180,0.9636),(0.7071,1.0177)$ \\
8 & 29 & $(1.0690,0.9449),(0.8452,0.9636)$ \\
15 & 29 & $(0.9636,0.9636),(1.1019,0.8018)$ \\
7 & 31 & $(1.1547,0.9129),(0.5774,0.9309)$ \\
16 & 31 & $(0.9309,0.9309),(1.0646,0.7746)$, \\
& & $(0.7303,1.0328)$ \\
\hline & &
\end{tabular}

of $n_{1}=11$ and $n_{2}=21$ points there exist two different Pareto combinations, both with a maximin distance equal to $d=0.7071$. For the $\left(n_{1}, n_{2}\right)$ pairs $(9,17)$ and $(10,19)$, the objective values of the Pareto nested designs are also equal (0.7906 and 0.7454 , respectively); however, the individual maximal distances of the second Pareto combination are smaller than the maximal distances of the (optimal) first combination $(1.0607<$ 1.1180 and $1.0000<1.0541$, respectively). The Pareto nested designs can also be found on the website http://www.spacefillingdesigns.nl.

\section{Higher dimensional nested designs}

\subsection{Enhanced stochastic evolutionary algorithm}

For dimensions higher than two and for larger values of $n_{1}$ and $n_{2}$, the above branch-and-bound algorithm to find nested LHDs which maximize $d$ becomes too time-consuming. In these cases, we can use heuristics to find nested approximate maximin LHDs, where "approximate" indicates that optimality is not guaranteed. One possible heuristic is the ESE algorithm of Jin et al. (2005). In Husslage et al. (2008), this algorithm obtains good results for approximate maximin LHDs. Furthermore, the algorithm is used in Viana et al. (2007) to generate space-filling LHDs. Although this algorithm was originally designed for non-nested designs, with some changes it is also applicable to nested designs. Before we look at these changes, we first give a short description of the original ESE algorithm. This description is based on the description given in Husslage et al. (2008).

The algorithm starts with an initial design and tries to find better designs by iteratively changing the current design. To determine if a new design is accepted, a threshold-based acceptance criterion is used. This criterion is controlled in the outer loop of the algorithm. In the inner loop of the algorithm new designs are explored.

The inner loop explores the design space as follows. At each iteration, first a dimension $k$ is selected. The algorithm then creates a fixed number of new designs by exchanging the $k^{\text {th }}$ coordinate value of two randomly chosen points of the current design. The new design with the largest separation distance is then compared to the current design using a threshold criterion. The criterion ensures that better designs are always accepted and that worse designs can be accepted with a certain probability depending on the threshold value. If the new design is accepted, it replaces the current design. This process is repeated until a certain stopping criterion is met.

The outer loop controls the threshold value. After the inner loop is completed, the outer loop determines how much improvement is made in the inner loop. If the amount of improvement is above a certain level, the algorithm starts an improving process in which it tries to rapidly find a local optimum. It does this by lowering the threshold value and thus accepting fewer deteriorations in the inner loop. If too little improvement is made, an exploration process is started which is intended to escape from a local optimum. The threshold value is first rapidly increased to move away from a local optimum and later slowly decreased to find better designs after moving away. The final design of the algorithm is the best design found during all iterations of the inner loop.

To use the ESE algorithm for nested designs, the step which needs to be changed most is the generation of new designs. When one point is selected from $X_{1}$ and the other from $X_{2} \backslash X_{1}$, exchanging the $k^{\text {th }}$ coordinate value can distort the nested grid-structure.

Figures 8 and 9 give an example where this distortion indeed occurs. The design in Fig. 9 is obtained by exchanging one coordinate value of two points in the lower left part of Fig. 8. As we require in each dimension that the first and last point should be in $X_{1}$, the new design is not a valid nested design. We could try to repair this by changing the assignment of the points to the sets $X_{1}$ and $X_{2}$. However, there exists no 


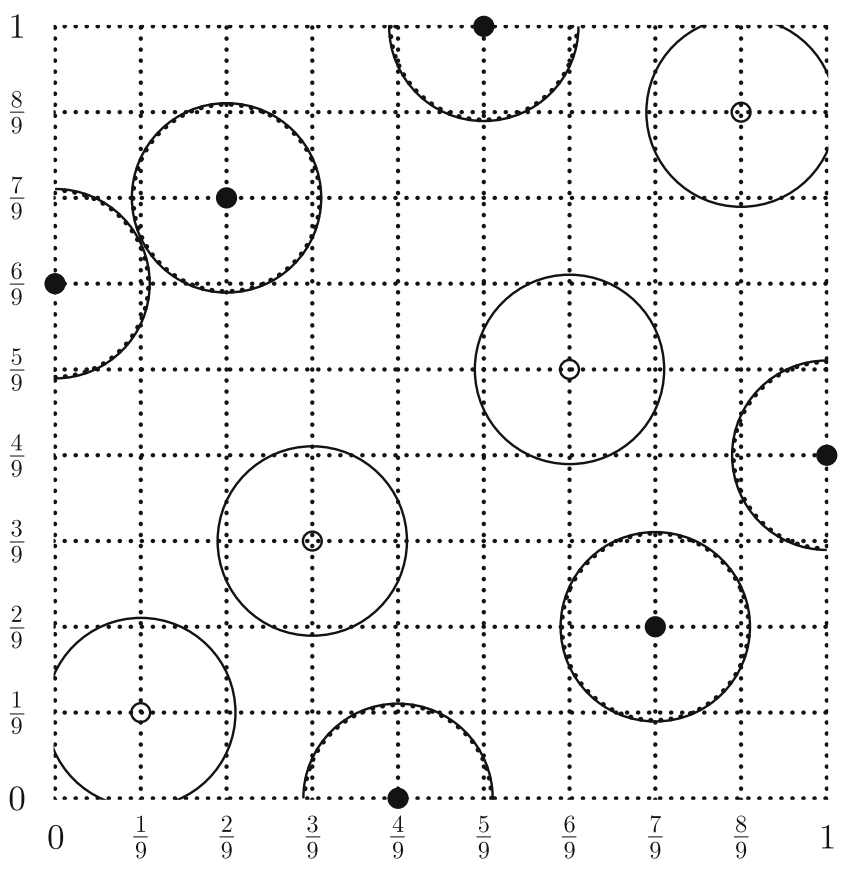

Fig. 8 A nested Latin hypercube design of $n_{1}=6$ and $n_{2}=10$ points; $d=d_{1}=0.3086$ and $d_{2}=0.5556$

assignment such that the invalid nested design in Fig. 9 becomes a valid nested LHD on a nested $n_{2}$-grid.

\subsection{Generating new designs}

The main problem which needs to be addressed by a new method for generating designs is the distortion of the chosen grid-structure. Fortunately, we can quite easily avoid this problem in the following way. Instead of randomly choosing two points from the complete set of points, we randomly choose two points from either $X_{1}$ or $X_{2} \backslash X_{1}$. By exchanging coordinate values between two points within the same set, the chosen grid-structure is always maintained.

We also want to take into account that the grids are not unique when $c_{2}$ is non-integer. For instance, when $n_{1}=6$ and $n_{2}=13$, it can be verified that there are 21 different two-dimensional nested $n_{2}$-grids, after accounting for reflection and rotational symmetry. In cases like these, the choice of a specific grid can affect the maximal attainable value of $d$. Therefore, we run the ESE algorithm for different initial grids and designs. Furthermore, besides the exchange of coordinate values, we also consider other transformations which are able to change a grid without distorting it.

Based on the above observations, we developed four different methods for generating new designs. We describe the most successful method in this section. A description of the other three methods and a comparison of all four methods can be found in Appendix A.

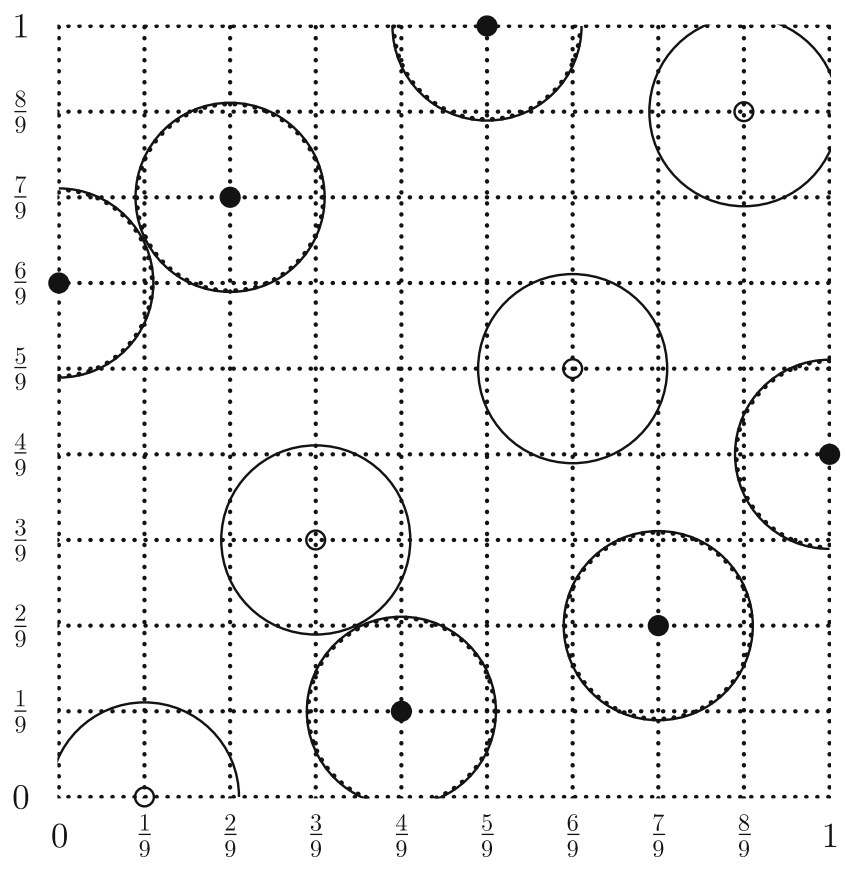

Fig. 9 A nested design obtained by exchanging one coordinate value between a point in $X_{1}$ and one point in $X_{2} \backslash X_{1}$

The most successful method method, called GROUPRAND, can change both the grid and the selected points of a design. Remember that for all types of grids, there must be either $\left\lfloor c_{2}\right\rfloor-1$ or $\left\lceil c_{2}\right\rceil-1$ $X_{2}$-coordinates between every pair of consecutive $X_{1}$ coordinates. By deciding between which pairs $\left\lfloor c_{2}\right\rfloor-1$ points are placed and between which pairs $\left\lceil c_{2}\right\rceil-1$ points are placed, we fix a grid. To change a grid without it becoming invalid, we thus have to change the assignment of $\left\lfloor c_{2}\right\rfloor-1$ and $\left\lceil c_{2}\right\rceil-1 \quad X_{2}$-points to the pairs of consecutive $X_{1}$-coordinates. This principle leads to the following definition of the GROUPRAND method.

The method GROUPRAND starts with randomly selecting a first point from $X_{2}$. If a point in $X_{1}$ is selected, we simply exchange two points in $X_{1}$. Otherwise, we select with equal probability to either exchange the selected point with another point in $X_{2} \backslash X_{1}$ or to perform a group-exchange. A group-exchange is performed by first selecting two pairs of consecutive $X_{1}$-points, i.e. $X_{1}$-points which have consecutive $X_{1}$ coordinates in the $k^{\text {th }}$ dimension. All $X_{2}$ points between a pair of consecutive $X_{1}$-points are now referred to as a group. Note that when $\left\lfloor c_{2}\right\rfloor=1$, a group can be empty. To generate a new design, we now switch the two groups. As both groups contain $\left\lfloor c_{2}\right\rfloor-1$ or $\left\lceil c_{2}\right\rceil-1$ points, this will result in a valid nested design. When the number of points in the groups differ, the exchange of the groups also changes the grid. Depending on the type of grid, the group exchange not only affects the position 


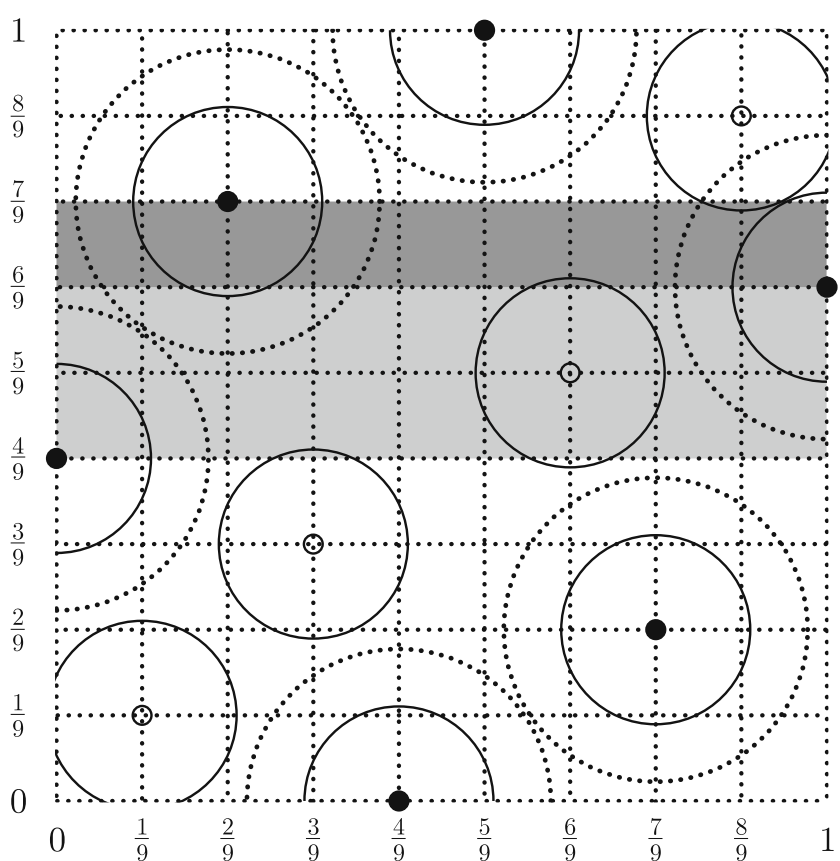

Fig. 10 A nested Latin hypercube design of $n_{1}=6$ and $n_{2}=10$ points; $d=d_{2}=0.7454$ and $d_{1}=0.8958$

of the points in the group but possibly also other points. Which and how other points are affected differs per type of grid, but is fairly straightforward to determine.

To illustrate the GROUPRAND method, we will use the design in Fig. 10 and take $k$ equal to 2, i.e. the dimension on the vertical axis. The two differently shaded areas in Fig. 10 now form two possible groups. Notice that the top group is empty as there are no $X_{2}$-points between the $X_{1}$-points. In Fig. 11, we see the result of exchanging the two groups. Because the groups are of different size, the grid has now changed. Comparing the values of $d$ shows that the design has improved and, in this case, is even optimal.

\section{Numerical results}

In the numerical comparison in this section, we consider nested designs of dimensions two up to ten. The two-dimensional nested maximin designs are obtained with the branch-and-bound algorithm described in Section 4.1. The three- and four-dimensional nested approximate maximin designs are obtained by performed ten runs of each variant of the ESE algorithm described in Section 5.2 and Appendix A. For dimensions five up to ten, we only used ten runs of the GROUPRAND algorithm. In this comparison, we consider the best obtained designs and all ten designs obtained with GROUPRAND. For each dimension and gridstructure, we ran the ESE algorithm for 65 differ-

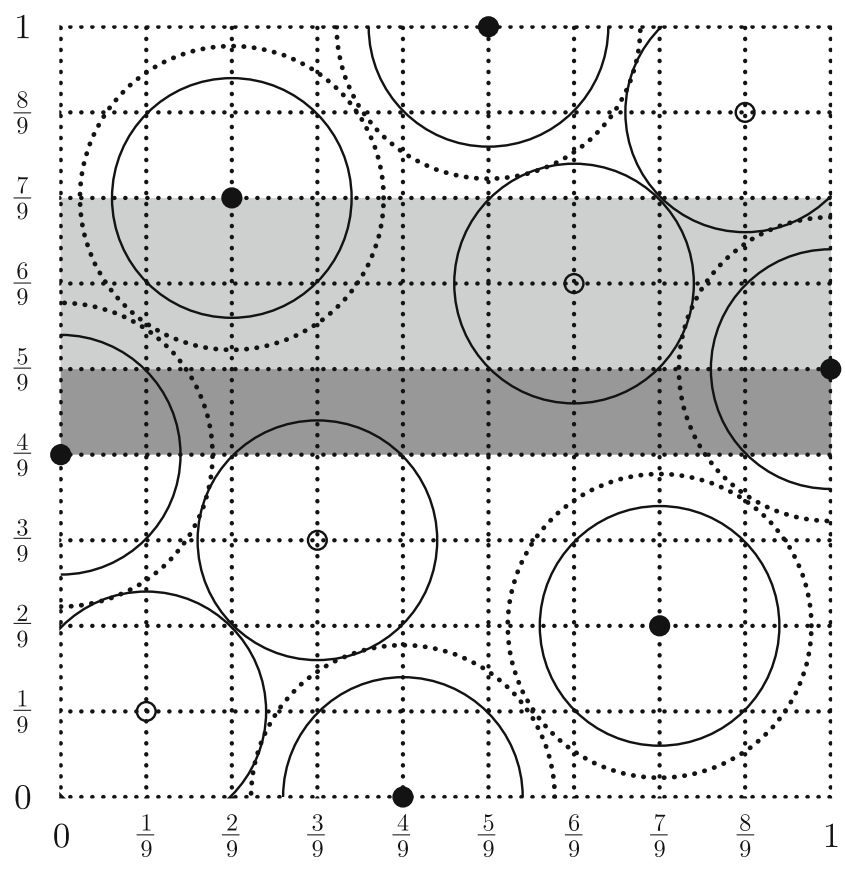

Fig. 11 A nested maximin Latin hypercube design of $n_{1}=6$ and $n_{2}=10$ points $d=d_{1}=0.8958$ and $d_{2}=0.9428$

ent pairs $\left(n_{1}, n_{2}\right)$ with $n_{1}=5,10, \ldots, 50$ and $n_{2}=n_{1}+$ $5, \ldots, 55,60$. The computations have been performed on PCs with a $2.8-\mathrm{GHz}$ Pentium D processor and the variants were implemented in Matlab 7.4 R2007a. More details on the computations can be found in Appendices A and C.

To get an impression of the calculation times, we determined the mean and average of the calculation times over all ten runs of the GROUPRAND algorithm (for each pair $\left(n_{1}, n_{2}\right)$ and dimension $m$ ). Table 2 contains the results for the nested $n_{1}$-grid and Table 13 in Appendix $\mathrm{C}$ contains the results for all three grids. When evaluating these results, we note that the calculation times are mainly influenced by two factors. Firstly, generating a new design and calculating $d$ becomes more time-consuming when $n_{2}$ or the dimension $m$ increases. Especially, updating the distance matrix containing the distances between all design points requires more time. Secondly, the total number of iterations of the outer loop can vary over different runs of the ESE algorithm. This is caused by our stopping criterion, which terminates the algorithm when no improvement has been found in the last 100 iterations of the outer loop.

The effect of $n_{2}$ is most apparent when we compare the calculation times for a fixed $n_{1}$. For some cases, especially with $n_{1}=5$, the calculation times are quite large. Note however, that once we have generated a good design for a certain pair $\left(n_{1}, n_{2}\right)$ in a certain dimension, we can use this design many times without 
Table 2 Mean and standard deviation of calculation times in minutes of GROUPRAND for the nested $n_{1}$-grid

\begin{tabular}{|c|c|c|c|c|c|c|c|c|c|}
\hline \multirow[t]{3}{*}{$n_{1}$} & \multirow[t]{3}{*}{$n_{2}$} & \multicolumn{8}{|c|}{ Calculation time } \\
\hline & & \multicolumn{4}{|c|}{ Mean } & \multicolumn{4}{|l|}{ Std } \\
\hline & & $\overline{4 d}$ & $6 \mathrm{~d}$ & $8 \mathrm{~d}$ & $10 \mathrm{~d}$ & $\overline{4 d}$ & $6 \mathrm{~d}$ & $8 \mathrm{~d}$ & $10 \mathrm{~d}$ \\
\hline$\overline{5}$ & 10 & 0.1 & 0.3 & 0.4 & 0.3 & 0.0 & 0.1 & 0.1 & 0.1 \\
\hline 5 & 20 & 1.6 & 4.3 & 4.9 & 3.6 & 1.2 & 1.8 & 1.7 & 1.4 \\
\hline 5 & 30 & 8.0 & 22.6 & 21.4 & 15.0 & 5.0 & 6.9 & 5.0 & 5.1 \\
\hline 5 & 40 & 19.2 & 40.6 & 51.5 & 23.7 & 14.8 & 5.7 & 9.6 & 3.4 \\
\hline 5 & 50 & 27.0 & 90.0 & 118.2 & 66.0 & 15.2 & 19.4 & 17.9 & 11.5 \\
\hline 5 & 60 & 26.4 & 176.8 & 216.5 & 118.5 & 5.4 & 47.7 & 30.7 & 16.3 \\
\hline 10 & 15 & 0.1 & 0.6 & 0.8 & 0.7 & 0.0 & 0.2 & 0.2 & 0.3 \\
\hline 10 & 20 & 0.3 & 1.5 & 2.6 & 2.1 & 0.1 & 0.5 & 1.1 & 0.8 \\
\hline 10 & 30 & 1.5 & 8.3 & 10.4 & 5.3 & 0.4 & 2.6 & 4.8 & 1.5 \\
\hline 10 & 40 & 4.4 & 13.3 & 18.2 & 7.4 & 1.4 & 4.9 & 7.7 & 1.2 \\
\hline 10 & 50 & 5.6 & 23.1 & 24.8 & 14.4 & 1.9 & 12.0 & 7.4 & 4.8 \\
\hline 10 & 60 & 7.0 & 31.2 & 37.8 & 23.1 & 4.2 & 7.4 & 10.3 & 4.9 \\
\hline 15 & 20 & 0.3 & 1.6 & 2.2 & 1.3 & 0.1 & 0.4 & 0.6 & 0.4 \\
\hline 15 & 30 & 1.2 & 6.6 & 5.9 & 4.5 & 0.4 & 2.3 & 1.6 & 1.5 \\
\hline 15 & 40 & 2.0 & 8.1 & 8.9 & 6.2 & 0.8 & 2.9 & 3.2 & 2.9 \\
\hline 15 & 50 & 3.4 & 13.2 & 15.8 & 8.6 & 1.5 & 4.8 & 7.4 & 2.5 \\
\hline 15 & 60 & 4.7 & 23.5 & 25.0 & 15.7 & 2.4 & 13.6 & 7.3 & 4.8 \\
\hline 20 & 25 & 0.6 & 3.1 & 3.1 & 3.4 & 0.2 & 1.6 & 1.1 & 1.6 \\
\hline 20 & 30 & 1.2 & 6.2 & 7.2 & 3.8 & 0.4 & 2.3 & 2.8 & 1.3 \\
\hline 20 & 40 & 2.1 & 7.9 & 8.8 & 4.4 & 0.5 & 2.3 & 3.1 & 1.3 \\
\hline 20 & 50 & 3.2 & 12.8 & 16.5 & 8.9 & 1.5 & 5.9 & 8.6 & 2.8 \\
\hline 20 & 60 & 3.0 & 20.3 & 17.9 & 11.7 & 1.2 & 7.9 & 6.8 & 2.8 \\
\hline 25 & 30 & 1.2 & 5.7 & 6.9 & 4.4 & 0.3 & 1.0 & 2.7 & 1.0 \\
\hline 25 & 40 & 2.4 & 9.2 & 10.3 & 8.2 & 0.6 & 2.7 & 3.0 & 3.5 \\
\hline 25 & 50 & 2.8 & 13.7 & 17.7 & 8.4 & 0.7 & 6.3 & 7.7 & 3.7 \\
\hline 25 & 60 & 5.4 & 19.3 & 25.6 & 22.0 & 2.7 & 9.1 & 9.3 & 6.4 \\
\hline 30 & 35 & 2.0 & 8.4 & 9.6 & 6.6 & 0.9 & 2.5 & 1.7 & 2.7 \\
\hline 30 & 40 & 2.6 & 10.4 & 13.4 & 10.6 & 0.7 & 3.1 & 5.3 & 3.0 \\
\hline 30 & 50 & 3.3 & 16.1 & 19.4 & 15.1 & 0.3 & 4.8 & 4.1 & 4.5 \\
\hline 30 & 60 & 5.8 & 21.3 & 27.8 & 18.3 & 1.8 & 6.5 & 11.4 & 8.7 \\
\hline 35 & 40 & 2.8 & 9.4 & 12.9 & 9.9 & 0.9 & 2.4 & 3.6 & 3.0 \\
\hline 35 & 50 & 4.3 & 18.9 & 24.6 & 20.2 & 0.7 & 6.1 & 5.7 & 6.9 \\
\hline 35 & 60 & 5.6 & 26.5 & 32.6 & 29.2 & 1.7 & 13.0 & 9.4 & 11.1 \\
\hline 40 & 45 & 6.1 & 16.0 & 16.2 & 13.9 & 2.8 & 6.6 & 7.3 & 3.8 \\
\hline 40 & 50 & 9.9 & 28.4 & 29.0 & 18.7 & 4.6 & 11.8 & 9.1 & 3.9 \\
\hline 40 & 60 & 6.0 & 30.5 & 42.5 & 33.2 & 2.9 & 7.7 & 10.4 & 10.0 \\
\hline 45 & 50 & 5.3 & 19.3 & 22.8 & 20.9 & 1.6 & 6.9 & 4.2 & 6.6 \\
\hline 45 & 60 & 9.0 & 40.6 & 52.8 & 35.8 & 3.0 & 10.4 & 17.4 & 15.5 \\
\hline 50 & 55 & 10.4 & 29.6 & 33.9 & 35.8 & 4.0 & 10.7 & 6.6 & 8.1 \\
\hline 50 & 60 & 13.0 & 40.4 & 48.4 & 30.9 & 6.9 & 10.3 & 17.8 & 12.5 \\
\hline
\end{tabular}

incurring these calculation times again. When we compare the times for different dimensions $m$, we see that the times generally do increase, but not as much as one might expect. For many pairs the times for 10 dimensions are even lower than for 8 dimensions. This result is probably caused by the second factor, i.e., the variance in number of iterations caused by the stopping criterion. When we consider a certain pair $\left(n_{1}, n_{2}\right)$ and dimension $m$, this factor is also the main cause for the variance of the calculation times.
Besides the calculation times, we are also interested in the performance of GROUPRAND in terms of the space-fillingness of the obtained nested designs. As there are no other results in literature for (approximatie) maximin nested designs, we cannot compare our results. Therefore, we decided to perform Monte Carlo simulations to get an impression of how the obtained designs are positioned in the objective function space. We performed these Monte Carlo simulations by randomly generating 10,000 nested designs for each gridstructure, pair $\left(n_{1}, n_{2}\right)$ and dimension $m$. We denote the mean, standard deviation and maximum of the $d$-values of these 10,000 designs by mean $m c, s t d_{m c}$ and max $_{m c}$, respectively. In Table 3 , we compare these values to $d_{b e s t}$, i.e., the $d$ value of the best obtained nested (approximate) maximin design. The first figure shows how many times $d_{b e s t}$ is larger than mean ${ }_{m c}$. The second figures also shows the difference between $d_{b e s t}$ and mean $_{m c}$, but now in terms of the number of standard deviations $s t d_{m c}$. The last figure compares $d_{b e s t}$ to $\max _{m c}$, i.e., the $d$-value of the best design found by the Monte Carlo simulation. All three figures show that $d_{b e s t}$ is considerably larger than mean ${ }_{m c}$ and $\max _{m c}$. The GROUPRAND algorithm is thus able to significantly improve the space-fillingness of the nested designs.

However, compared to non-nested designs of sizes $n_{1}$ and $n_{2}$, we expect a lower space-fillingness because of the additional condition that $X_{1}$ and $X_{2}$ should be nested. Therefore, we also evaluate the loss of space-fillingness by using nested instead of non-nested designs. To determine this, we compare the $d_{1}$ - and $d_{2}$-values of the nested (approximate) maximin designs to the scaled separation distances of the (approximate) maximin LHDs of the same size. For a pair $\left(n_{1}, n_{2}\right)$, we denote the first distances by $d_{1}\left(n_{1}, n_{2}\right)$ and $d_{2}\left(n_{1}, n_{2}\right)$ and the latter distances by $d\left(n_{1}\right)$ and $d\left(n_{2}\right)$. For $d\left(n_{1}\right)$ and $d\left(n_{2}\right)$, we use the best known (approximate) maximin LHDs available on http://www. spacefillingdesigns.nl (December 2008). We now define the percentage loss in scaled separation distance as:

$l_{j}\left(n_{1}, n_{2}\right):=\left(d_{j}\left(n_{1}, n_{2}\right)-d\left(n_{j}\right)\right) / d\left(n_{j}\right), j=1,2$.

Table 4 represents the averages and the ranges of the percentage losses for the best nested (approximate) maximin designs. The average and range are both taken over all evaluated $\left(n_{1}, n_{2}\right)$-pairs. Note that for two dimensions, we evaluated different pairs than for the other dimensions. When we consider the twodimensional results, we see that the $n_{2}$-grid on average gives the best space-fillingness for both designs $X_{1}$ and $X_{2}$. For the higher dimensions, the averages and ranges are more similar, but the nested $n_{1}$-grid 
Table 3 Comparison of $d_{b e s t}$ to the $d$ values of the Monte Carlo simulation

\begin{tabular}{|c|c|c|c|c|c|c|c|c|}
\hline & \multicolumn{8}{|c|}{ Average over all $\left(n_{1}, n_{2}\right)$-pairs } \\
\hline & $3 d$ & $4 d$ & $5 d$ & $6 \mathrm{~d}$ & $7 \mathrm{~d}$ & $8 \mathrm{~d}$ & $9 \mathrm{~d}$ & $10 \mathrm{~d}$ \\
\hline \multicolumn{9}{|l|}{ Nested $n_{1}$-grid } \\
\hline$d_{\text {best }} /$ mean $_{m c}$ & 4.0 & 4.5 & 4.9 & 5.3 & 5.6 & 5.9 & 6.2 & 6.4 \\
\hline$\left(d_{b e s t}-\right.$ mean $\left._{m c}\right) / s t d_{m c}$ & 10.2 & 11.9 & 13.4 & 14.6 & 15.7 & 16.7 & 17.6 & 18.4 \\
\hline$\left(d_{b e s t}-\max _{m c}\right) / s t d_{m c}$ & 6.4 & 8.2 & 9.6 & 10.9 & 12.0 & 13.0 & 13.9 & 14.7 \\
\hline \multicolumn{9}{|l|}{ Nested $n_{2}$-grid } \\
\hline$d_{\text {best }} /$ mean $_{m c}$ & 3.9 & 4.3 & 4.7 & 5.1 & 5.4 & 5.7 & 5.9 & 6.1 \\
\hline$\left(d_{\text {best }}-\right.$ mean $\left._{m c}\right) / s t d_{m c}$ & 10.2 & 11.8 & 13.2 & 14.4 & 15.5 & 16.5 & 17.3 & 18.2 \\
\hline$\left(d_{b e s t}-\max _{m c}\right) / s t d_{m c}$ & 6.4 & 8.0 & 9.4 & 10.6 & 11.7 & 12.7 & 13.5 & 14.4 \\
\hline \multicolumn{9}{|l|}{ Nested maximin axes } \\
\hline$d_{\text {best }} /$ mean $_{m c}$ & 3.9 & 4.4 & 4.8 & 5.1 & 5.4 & 5.7 & 6.0 & 6.2 \\
\hline$\left(d_{\text {best }}-\right.$ mean $\left._{m c}\right) / s t d_{m c}$ & 10.2 & 11.8 & 13.2 & 14.4 & 15.5 & 16.5 & 17.4 & 18.2 \\
\hline$\left(d_{b e s t}-\max _{m c}\right) / s t d_{m c}$ & 6.4 & 8.0 & 9.4 & 10.6 & 11.7 & 12.7 & 13.6 & 14.4 \\
\hline
\end{tabular}

small number of runs of the GROUPRAND algorithm is sufficient to determine a good nested approximate maximin design. The relatively high standard deviation for cases with $n_{1}=5$ indicates that for these cases it is beneficial to perform more runs of the GROUPRAND algorithm. When we compare the results for different dimensions, there is no real pattern in the standard deviations.

Besides the performance of GROUPRAND, we are also interested in the performance of the different gridstructures. As can already be seen from the results in Tables 10, 11, and 12, which grid gives the best separation distances depends on the particular pair $\left(n_{1}, n_{2}\right)$ and dimension. To get an idea of which grid-structures perform well in general, we present the percentages of pairs $\left(n_{1}, n_{2}\right)$, with $c_{2} \notin \mathbb{N}$, for which a grid type performs best on a particular distance in Table 6. We do not consider the pairs with $c_{2} \in \mathbb{N}$ because for these pairs all grids are equal.

Not surprisingly, the grids with the lowest average loss in Table 4 also have the highest percentage of pairs for which they perform best. However, there is still a considerable percentage of pairs where one of the other two grids perform better. It thus strongly depends on
Table 4 Average and range of percentage loss $l_{j}\left(n_{1}, n_{2}\right)$ for the best nested (approximate) maximin designs over all evaluated $\left(n_{1}, n_{2}\right)$-pairs

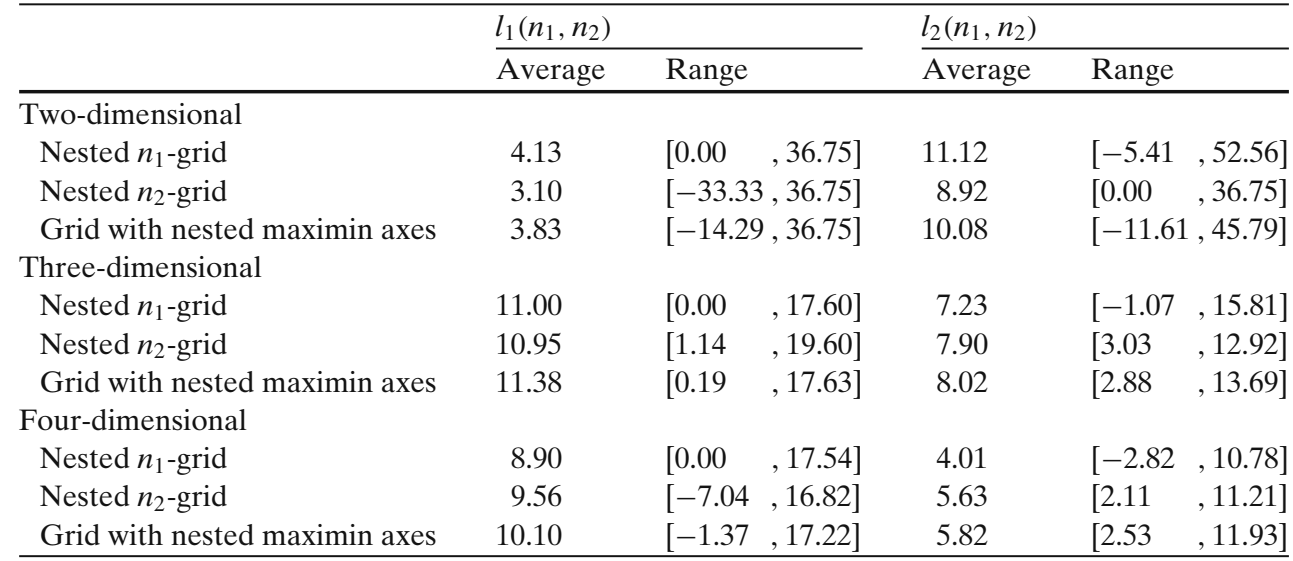


Table 5 Mean and standard deviation of percentage loss $l_{1}\left(n_{1}, n_{2}\right)$ of GROUPRAND for the nested $n_{1}$-grid

\begin{tabular}{|c|c|c|c|c|c|c|c|c|c|}
\hline \multirow[t]{3}{*}{$n_{1}$} & \multirow[t]{3}{*}{$n_{2}$} & \multicolumn{8}{|c|}{$l_{1}\left(n_{1}, n_{2}\right)$} \\
\hline & & \multicolumn{4}{|l|}{ Mean } & \multicolumn{4}{|l|}{ Std } \\
\hline & & $4 d$ & $6 \mathrm{~d}$ & $8 \mathrm{~d}$ & $10 \mathrm{~d}$ & $4 d$ & $6 \mathrm{~d}$ & $8 \mathrm{~d}$ & $10 \mathrm{~d}$ \\
\hline 5 & 10 & 6.06 & 10.21 & 10.85 & 11.49 & 6.80 & 3.71 & 1.85 & 2.65 \\
\hline 5 & 20 & 9.64 & 12.70 & 15.97 & 12.59 & 6.23 & 3.03 & 3.89 & 4.74 \\
\hline 5 & 30 & 9.19 & 13.17 & 18.20 & 12.78 & 4.64 & 4.30 & 3.02 & 3.64 \\
\hline 5 & 40 & 10.61 & 15.57 & 15.52 & 15.31 & 3.12 & 4.66 & 3.89 & 5.58 \\
\hline 5 & 50 & 10.42 & 15.57 & 14.33 & 12.98 & 6.82 & 4.66 & 3.41 & 5.52 \\
\hline 5 & 60 & 9.36 & 16.09 & 13.93 & 11.09 & 7.57 & 6.10 & 4.29 & 3.96 \\
\hline 10 & 15 & 11.92 & 6.97 & 5.09 & 3.90 & 1.48 & 0.95 & 0.59 & 0.70 \\
\hline 10 & 20 & 16.24 & 13.31 & 10.24 & 9.39 & 2.13 & 1.11 & 1.68 & 1.46 \\
\hline 10 & 30 & 17.69 & 14.43 & 12.09 & 12.05 & 2.33 & 1.00 & 1.22 & 0.88 \\
\hline 10 & 40 & 17.93 & 15.39 & 13.38 & 13.33 & 2.30 & 1.56 & 1.08 & 0.56 \\
\hline 10 & 50 & 18.77 & 16.86 & 14.60 & 13.97 & 1.54 & 1.50 & 0.69 & 1.03 \\
\hline 10 & 60 & 20.91 & 17.44 & 15.10 & 14.54 & 2.20 & 1.38 & 1.47 & 0.80 \\
\hline 15 & 20 & 8.64 & 4.06 & 5.65 & 3.43 & 1.01 & 0.91 & 1.36 & 1.17 \\
\hline 15 & 30 & 15.01 & 9.42 & 10.90 & 7.69 & 0.99 & 0.72 & 1.04 & 0.74 \\
\hline 15 & 40 & 15.74 & 11.25 & 11.16 & 8.60 & 0.87 & 1.78 & 0.64 & 0.69 \\
\hline 15 & 50 & 16.42 & 12.60 & 13.26 & 10.82 & 1.65 & 1.04 & 1.37 & 0.49 \\
\hline 15 & 60 & 17.43 & 13.25 & 14.38 & 11.74 & 1.54 & 0.71 & 0.87 & 0.77 \\
\hline 20 & 25 & 8.67 & 4.44 & 2.56 & 6.42 & 1.38 & 1.09 & 0.68 & 1.06 \\
\hline 20 & 30 & 9.91 & 4.95 & 3.12 & 7.61 & 0.95 & 0.83 & 0.90 & 0.73 \\
\hline 20 & 40 & 14.31 & 10.68 & 7.67 & 11.41 & 0.64 & 1.04 & 0.69 & 0.43 \\
\hline 20 & 50 & 14.65 & 10.37 & 6.36 & 10.06 & 0.77 & 1.48 & 1.06 & 0.54 \\
\hline 20 & 60 & 17.58 & 12.41 & 10.23 & 13.37 & 1.58 & 0.94 & 0.67 & 0.49 \\
\hline 25 & 30 & 4.88 & 3.29 & 2.30 & 2.37 & 1.39 & 0.48 & 0.51 & 0.37 \\
\hline 25 & 40 & 8.91 & 5.53 & 3.44 & 2.96 & 0.89 & 0.51 & 0.48 & 0.51 \\
\hline 25 & 50 & 13.07 & 10.28 & 7.14 & 7.11 & 0.60 & 1.14 & 0.80 & 0.79 \\
\hline 25 & 60 & 12.73 & 9.61 & 5.73 & 4.57 & 1.46 & 1.49 & 0.97 & 0.50 \\
\hline 30 & 35 & 3.99 & 2.68 & 3.44 & 2.35 & 1.00 & 0.72 & 0.48 & 0.45 \\
\hline 30 & 40 & 5.74 & 3.66 & 3.77 & 2.53 & 0.53 & 0.57 & 0.56 & 0.50 \\
\hline 30 & 50 & 9.80 & 5.93 & 4.83 & 3.06 & 0.32 & 0.50 & 0.43 & 0.28 \\
\hline 30 & 60 & 11.72 & 9.51 & 8.64 & 6.58 & 0.85 & 0.51 & 0.93 & 0.52 \\
\hline 35 & 40 & 3.42 & 3.06 & 2.69 & 2.21 & 1.00 & 0.89 & 0.44 & 0.40 \\
\hline 35 & 50 & 6.20 & 3.99 & 3.00 & 2.51 & 0.67 & 1.01 & 0.43 & 0.35 \\
\hline 35 & 60 & 9.35 & 6.46 & 4.63 & 3.40 & 0.94 & 0.59 & 0.58 & 0.48 \\
\hline 40 & 45 & 2.31 & 3.66 & 2.86 & 3.99 & 0.89 & 0.64 & 0.57 & 0.33 \\
\hline 40 & 50 & 3.01 & 4.07 & 3.44 & 4.45 & 0.96 & 0.90 & 1.47 & 0.20 \\
\hline 40 & 60 & 6.01 & 5.34 & 3.95 & 4.56 & 0.75 & 0.67 & 1.23 & 0.31 \\
\hline 45 & 50 & 2.43 & 3.59 & 1.98 & 1.61 & 0.71 & 0.60 & 0.43 & 0.30 \\
\hline 45 & 60 & 4.30 & 4.41 & 2.24 & 2.13 & 0.36 & 0.45 & 0.60 & 0.39 \\
\hline 50 & 55 & 2.48 & 1.41 & 1.56 & 1.71 & 0.91 & 0.59 & 0.32 & 0.32 \\
\hline 50 & 60 & 3.17 & 1.72 & 2.02 & 2.49 & 0.62 & 0.62 & 0.62 & 0.55 \\
\hline
\end{tabular}

the particular pair $\left(n_{1}, n_{2}\right)$ which grid to choose based on the separation distances.

Furthermore, in many practical situations, the values of $n_{1}$ and $n_{2}$ are not fixed which leaves some freedom to change these values. In those situations, we can thus also consider nested designs where $n_{1}$ and $n_{2}$ are slightly lower or higher. Let us, for example, consider the two-dimensional designs with $n_{1}=5$ and $n_{2}=10$. In Table 7, we compare the losses of these designs to the losses of the designs with $n_{1}=6$ and $n_{2}=10$. The comparison shows that all losses either reduce or stay
Table 6 Percentage of the $\left(n_{1}, n_{2}\right)$-pairs, with $c_{2} \notin \mathbb{N}$, for which a particular grid type performs best on $d, d_{1}$, or $d_{2}$

\begin{tabular}{lccc}
\hline & \multicolumn{3}{c}{ Percentage best designs } \\
\cline { 2 - 4 } & $\mathrm{d}$ & $\mathrm{d}_{1}$ & $\mathrm{~d}_{2}$ \\
\hline Two-dimensional & 17 & 36 & 16 \\
$\quad$ Nested $n_{1}$-grid & 67 & 56 & 72 \\
Nested $n_{2}$-grid & 16 & 11 & 13 \\
Grid with nested maximin axes & & & \\
Three-dimensional & 52 & 45 & 53 \\
$\quad$ Nested $n_{1}$-grid & 19 & 37 & 21 \\
Nested $n_{2}$-grid & 29 & 18 & 26 \\
Grid with nested maximin axes & & & \\
Four-dimensional & 69 & 71 & 66 \\
$\quad$ Nested $n_{1}$-grid & 24 & 18 & 26 \\
Nested $n_{2}$-grid & 8 & 11 & 10 \\
Grid with nested maximin axes & &
\end{tabular}

the same. Choosing $n_{1}$ equal to 6 instead of 5 thus seems to be a better choice in terms of space-fillingness.

As mentioned in Section 3.4, the choice for a specific grid or $\left(n_{1}, n_{2}\right)$-pair does not only depend on the spacefillingness. The users preferences and the reason why a nested design is used also affect the choice for a particular grid-structure.

\section{Concluding remarks}

A nested design consists of two separate designs, one being a subset of the other. Using these nested designs, instead of traditional designs of computer experiments, is useful when dealing with training and test sets, models with different levels of accuracy, linking parameters, or sequential evaluations, because nested designs are able to capture the dependencies between the two black-box functions or evaluation stages (with respect to the design parameters). This paper focuses on constructing nested (approximate) maximin Latin hypercube designs in two and higher dimensions. The maximin criterion is used to find space-filling nested designs, i.e., designs with the design points spread over the entire design space. By choosing the design points on a grid, we ensure non-collapsingness, i.e., no two design points will have the same coordinate values. We distinguish between three types of grids: a nested $n_{1}$ grid, a nested $n_{2}$-grid, and a grid with nested maximin

Table 7 Example of reduction in percentage loss $l_{j}\left(n_{1}, n_{2}\right)$ by choosing different value for $n_{1}$

\begin{tabular}{lllrc}
\hline Two-dimensional & $l_{1}(5,10)$ & $l_{2}(5,10)$ & $l_{1}(6,10)$ & $l_{2}(6,10)$ \\
\hline Nested $n_{1}$-grid & 36.75 & 16.15 & 0.00 & 10.00 \\
Nested $n_{2}$-grid & 28.34 & 10.56 & 10.42 & 10.56 \\
Grid with nested & 31.20 & 12.28 & 2.02 & 8.17 \\
$\quad$ maximin axes & & & & \\
\hline
\end{tabular}


axes. Which grid to use is mainly depends on the application under consideration and the user's preferences. For two-dimensional designs, a branch-and-bound algorithm is used to obtain nested maximin designs for all grids and for values of $n_{2}$ up to 15 . In the special case where $n_{1}-1$ is a divisor of $n_{2}-1$, maximin distances up to $n_{2}=32$ are provided.

For dimensions higher than two, determining nested LHDs which maximize $d$ becomes too time-consuming. Instead, we determine nested approximate maximin LHDs which do not guarantee optimality of $d$. To determine these designs, we considered four variants of the ESE algorithm. We mainly focused our attention on the GROUPRAND method as this method performed best in a comparison of three- and four-dimensional designs. Using this method, designs for dimensions five up to ten and for up to 100 design points were obtained. Note that all variants of the ESE algorithms can also be used for higher dimensions and larger numbers of points. When the number of points or dimensions increases, changing the design and calculating the new value for $d$ will become more time-consuming. Comparing the calculation times over different dimensions shows that the times indeed do increase, but not as much as one might expect. A method to compensate the increase in calculation time could be to perform fewer iterations of the inner loop of the ESE algorithm, although this might reduce the quality of the final nested design.

Besides comparing the different variants, we also studied the performance using Monte Carlo simulation. The results of this simulation show that the ESE algorithm is able to significantly improve the spacefillingness of the nested designs. Furthermore, we considered the percentage loss in space-fillingness by using nested designs instead of non-nested designs. The results show that the nested $n_{2}$-grid in general gives the smallest losses in two dimensions and the nested $n_{1}$-grid in higher dimensions. We also noted that we can reduce the amount of loss by choosing slightly different values for $n_{1}$ and $n_{2}$.

We remark that the objective $d=\min \left\{d_{1}, d_{2}\right\}$ used in this paper is only one way of combining the separation distances of $X_{1}$ and $X_{2}$. As mentioned in the introduction, alternative scaling factors and formulations are possible. Taking the weighted sum of both objectives instead of the minimum would be a possible alternative objective. When using this objective, the branch-andbound and ESE algorithms can still be used with little or no adjustments. Dealing with maximizing $d_{1}$ and $d_{2}$ as a bi-objective optimization problem is another possibility. In that case, different Pareto optimal nested designs could be found by using the weighted sum objective with various scaling factors.
Furthermore, we could also change the number of designs we want to nest. For instance in multi-fidelity modeling, we could come across models with more than two levels of accuracy. In these situations, we can use a nested design consisting of more than two designs. Note that for one-dimensional designs, Van Dam et al. (2009a) already consider optimizing the maximin criterion for these nested designs. To obtain results for higher dimensions, we could try to extend the branch-and-bound and ESE methods. The three main challenges would then be the following. Firstly, we have to find suitable grid-structures such that each design satisfies the LHD-structure as much as possible. This will become more difficult when we want to nest more designs. To avoid this problem, we could initially only consider nested designs for which all designs can satisfy the LHD-structure. When nesting three designs $X_{1} \subset$ $X_{2} \subset X_{3}$ with $n_{1}, n_{2}$ and $n_{3}$ design points, this would be possible if both $\frac{n_{2}-1}{n_{1}-1}$ and $\frac{n_{3}-1}{n_{2}-1}$ are integer. Secondly, we should decide on a criterion or method to achieve good space-fillingness for all the designs. Although we also need to make this decision for two sets, this decision will become more difficult when more sets have to be nested. Thirdly, when using the ESE algorithm, the methods for generating new designs should not distort the grid-structure. Depending on the grid-structure, we should determine whether the methods presented in this paper are still applicable or if they should be adjusted. When a suitable grid and method is determined and a single objective is used for space-fillingness, the ESE method in this paper can be used to generate nested designs with more than two designs.

Acknowledgement The authors would like to thank the referees for their valuable comments.

Open Access This article is distributed under the terms of the Creative Commons Attribution Noncommercial License which permits any noncommercial use, distribution, and reproduction in any medium, provided the original author(s) and source are credited.

\section{Appendix A: Variants of ESE algorithm}

Besides the GROUPRAND method, we developed and tested three other methods which differ in two aspects. Firstly, they differ in the method used to choose between $X_{1}$ and $X_{2} \backslash X_{1}$. In GROUPRAND random selection is used, but as we aim to maximize $\min \left\{d_{1}, d_{2}\right\}$, we could also base our choice on whether $d_{1}$ or $d_{2}$ is smallest. When $d_{1}$ is smallest, selecting two points from $X_{2} \backslash X_{1}$ might not be very useful as their positions do not directly influence the value of $d_{1}$. The value of $d_{2}$, 
on the other hand, does depend on the positions of all points and therefore both sets are relevant when $d_{2}$ is smallest.

Secondly, the methods differ in the possible exchanges. To test the effect of exchanging groups, we also tested methods which only allow the exchange of points. These methods might be faster as a group exchange is more difficult than a exchange of points. However, a drawback of not using group exchanges is that the initial grid cannot change.

As we have two options for each of the above aspects, we developed the following four methods for generating new designs. The method POINTRAND starts with randomly selecting a point from $X_{2}$. Depending on whether this point is in $X_{1}$ or not, we select a second point from $X_{1}$ or $X_{2} \backslash X_{1}$, respectively. This simple method is probably closest to the method of the original ESE algorithm. However, it does not take into account the values of $d_{1}$ and $d_{2}$ and is not able to change the grid.

The method POINTDMIN is similar to POINTRAND but does take the values of $d_{1}$ and $d_{2}$ into account. When $d_{2}$ is smallest, the method works in exactly the same way as POINTRAND. However when $d_{1}$ is smallest, only points from $X_{1}$ can be chosen as they are the only ones affecting $d_{1}$.

The third and fourth method, GROUPRAND and GROUPDMIN, are similar to POINTRAND and GROUPRAND, respectively. The only difference is that these two methods also allow group exchanges as described in Section 5.2.

To compare the four different variants of the ESE algorithm, we generated three- and four-dimensional nested designs with $n_{1}=5,10, \ldots, 50$ and $n_{2}=n_{1}+$ $5, \ldots, 55,60$ for each of the three grid-structures. We thus consider 65 different pairs $\left(n_{1}, n_{2}\right)$ for each dimension and grid-structure.

As the grid is not unique when $c_{2} \notin \mathbb{N}$, which grid we select might affect the space-fillingness of the final design. For each combination of $n_{1}, n_{2}$, dimension, and grid-structure, we therefore ran the ESE algorithm ten times with a different grid and initial design. For these ten runs, we tried two types of initial grids and designs: random and diagonal. For the first type, we randomly select a grid and design satisfying the restrictions of the chosen grid-structure. The second type starts with a diagonal design, where each design point has the same value for all coordinates. However, the results did not indicate a significant effect of the chosen type on the space-fillingness of the final design. Also the calculation times of the ESE algorithm did not significantly differ. Therefore, we do not make a distinction between these two types in the rest of this paper.

Using the best results of the ten runs of the ESE algorithm, we determine for each $\left(n_{1}, n_{2}\right)$-pair which method(s) obtained a best design. In Tables 11 and 12 of Appendix B, the separation distances of the best generated designs are given for each of the three gridstructures. The percentage of the $65\left(n_{1}, n_{2}\right)$-pairs for which a certain method performs best are presented in Table 8. Note that the sum of the percentages per row is larger than $100 \%$, because for some cases, a best design is found by multiple variants of the ESE algorithm. Due to the same reason, we cannot take the sum of two columns to determine the combined performance of two methods. When we study the results, we see that the two RAND methods find the best design for most cases. One reason for the relative poor performance of the DMIN methods could be that the number of neighbor designs is smaller. With neighbor designs, we mean all designs which can be obtained by making one possible change to the current design. When $d_{1}<d_{2}$, a DMIN method produces less neighbor designs than a RAND method, because the DMIN methods allow fewer possible changes. This can make it more difficult for a DMIN method to escape from a local minimum, which could result in a worse performance. Of the two RAND methods, GROUPRAND performs the best for most cases. This indicates that the possibility to change the grid indeed improves the performance of the ESE algorithm. Based on these results, we decided to use both RAND methods to obtain nested approximate maximin designs for dimensions five up to ten. However, in this paper we will only discuss the results of GROUPRAND.

Table 8 Percentage of the $\left(n_{1}, n_{2}\right)$-pairs for which a certain variant of the ESE algorithm finds a best design

\begin{tabular}{llllll}
\hline & RAND & & & DMIN & \\
\cline { 5 - 6 } & GROUP & POINT & & GROUP & POINT \\
\hline Three-dimensional & & & & & \\
Nested $n_{1}$-grid & 60 & 37 & 12 & 17 \\
Nested $n_{2}$-grid & 63 & 34 & 17 & 17 \\
Grid with nested & 55 & 23 & 6 & 17 \\
$\quad$ maximin axes & & & & \\
Four-dimensional & & & & \\
$\quad$ Nested $n_{1}$-grid & 66 & 26 & 2 & 11 \\
Nested $n_{2}$-grid & 57 & 29 & 6 & 15 \\
Grid with nested & 49 & 31 & 9 & 11 \\
$\quad$ maximin axes & & & &
\end{tabular}


Appendix B: Values of $\boldsymbol{d}$ for best nested (approximate) maximin designs

Table 9 provides the maximin distances for nested maximin Latin hypercube designs in two dimensions when $n_{1}-1$ is a divisor of $n_{2}-1$, i.e., $c_{2} \in$ $\mathbb{N}$, and for $n_{2} \leq 32$. For $n_{2}$ up to 15 , and $c_{2} \notin \mathbb{N}$, Table 10 provides the maximin distances for the two-dimensional nested maximin designs for all three grid-structures. Tables 11 and 12 provide the separation distances of three- and four-dimensional nested approximate maximin designs with $n_{1}=5,10, \ldots, 50$ and $n_{2}=n_{1}+5, \ldots, 55,60$ for all three grid-structures. Besides these distances, all tables also contain the scaled separation distances $d\left(n_{1}\right)$ and $d\left(n_{2}\right)$ of the approximate maximin LHDs available on http://www. spacefillingdesigns.nl (December 2008). The nested approximate maximin designs for dimensions five up to ten can also be found on this website.

Table 9 Maximin distances for two-dimensional nested maximin Latin hypercube designs; $c_{2} \in \mathbb{N}$

\begin{tabular}{lllllll}
\hline$n_{1}$ & $n_{2}$ & $d\left(n_{1}\right)$ & $d\left(n_{2}\right)$ & $d$ & $d_{1}$ & $d_{2}$ \\
\hline 2 & 3 & 1.4142 & 1.0000 & 1.0000 & 1.4142 & 1.0000 \\
2 & 4 & 1.4142 & 1.2910 & 0.8165 & 1.4142 & 0.8165 \\
2 & 5 & 1.4142 & 1.1180 & 0.7071 & 1.4142 & 0.7071 \\
3 & 5 & 1.0000 & 1.1180 & 0.7071 & 1.0000 & 0.7071 \\
2 & 6 & 1.4142 & 1.0000 & 1.0000 & 1.4142 & 1.0000 \\
2 & 7 & 1.4142 & 1.1547 & 0.9129 & 1.4142 & 0.9129 \\
3 & 7 & 1.0000 & 1.1547 & 0.9129 & 1.0000 & 0.9129 \\
4 & 7 & 1.2910 & 1.1547 & 0.8165 & 0.8165 & 1.1547 \\
2 & 8 & 1.4142 & 1.0690 & 0.8452 & 1.4142 & 0.8452 \\
2 & 9 & 1.4142 & 1.1180 & 1.0000 & 1.4142 & 1.0000 \\
3 & 9 & 1.0000 & 1.1180 & 1.0000 & 1.0000 & 1.0000 \\
5 & 9 & 1.1180 & 1.1180 & 1.1180 & 1.1180 & 1.1180 \\
2 & 10 & 1.4142 & 1.0541 & 0.9428 & 1.4142 & 0.9428 \\
4 & 10 & 1.2910 & 1.0541 & 0.8165 & 0.8165 & 0.9428 \\
2 & 11 & 1.4142 & 1.0000 & 1.0000 & 1.4142 & 1.0000 \\
3 & 11 & 1.0000 & 1.0000 & 1.0000 & 1.0000 & 1.0000 \\
6 & 11 & 1.0000 & 1.0000 & 1.0000 & 1.0000 & 1.0000 \\
2 & 12 & 1.4142 & 1.0871 & 0.9535 & 1.4142 & 0.9535 \\
2 & 13 & 1.4142 & 1.0408 & 0.9129 & 1.4142 & 0.9129 \\
3 & 13 & 1.0000 & 1.0408 & 0.9129 & 1.0000 & 0.9129 \\
4 & 13 & 1.2910 & 1.0408 & 0.9129 & 1.2910 & 0.9129 \\
5 & 13 & 1.1180 & 1.0408 & 0.8165 & 1.1180 & 0.8165 \\
7 & 13 & 1.1547 & 1.0408 & 0.9129 & 1.1547 & 0.9129 \\
2 & 14 & 1.4142 & 1.1435 & 1.0000 & 1.4142 & 1.0000 \\
2 & 15 & 1.4142 & 1.1019 & 0.9636 & 1.4142 & 0.9636 \\
3 & 15 & 1.0000 & 1.1019 & 0.8452 & 1.0000 & 0.8452 \\
8 & 15 & 1.0690 & 1.1019 & 0.8452 & 1.0690 & 0.8452 \\
\hline & & & & & &
\end{tabular}

Table 9 (continued)

\begin{tabular}{|c|c|c|c|c|c|c|}
\hline$\underline{n_{1}}$ & $n_{2}$ & $d\left(n_{1}\right)$ & $d\left(n_{2}\right)$ & $d$ & $d_{1}$ & $d_{2}$ \\
\hline 2 & 16 & 1.4142 & 1.0646 & 1.0646 & 1.4142 & 1.0646 \\
\hline 4 & 16 & 1.2910 & 1.0646 & 0.9309 & 1.2910 & 0.9309 \\
\hline 6 & 16 & 1.0000 & 1.0646 & 0.7303 & 1.0000 & 0.7303 \\
\hline 2 & 17 & 1.4142 & 1.0607 & 1.0308 & 1.4142 & 1.0308 \\
\hline 3 & 17 & 1.0000 & 1.0607 & 1.0000 & 1.0000 & 1.0308 \\
\hline 5 & 17 & 1.1180 & 1.0607 & 0.9014 & 1.1180 & 0.9014 \\
\hline 9 & 17 & 1.1180 & 1.0607 & 0.7906 & 1.1180 & 0.7906 \\
\hline 2 & 18 & 1.4142 & 1.0290 & 1.0000 & 1.4142 & 1.0000 \\
\hline 2 & 19 & 1.4142 & 1.0000 & 1.0000 & 1.4142 & 1.0000 \\
\hline 3 & 19 & 1.0000 & 1.0000 & 1.0000 & 1.0000 & 1.0000 \\
\hline 4 & 19 & 1.2910 & 1.0000 & 0.9718 & 1.2910 & 0.9718 \\
\hline 7 & 19 & 1.1547 & 1.0000 & 0.9718 & 1.1547 & 0.9718 \\
\hline 10 & 19 & 1.0541 & 1.0000 & 0.7454 & 1.0541 & 0.7454 \\
\hline 2 & 20 & 1.4142 & 0.9733 & 0.9733 & 1.4142 & 0.9733 \\
\hline 2 & 21 & 1.4142 & 1.0000 & 0.9487 & 1.4142 & 0.9487 \\
\hline 3 & 21 & 1.0000 & 1.0000 & 0.9487 & 1.0000 & 0.9487 \\
\hline 5 & 21 & 1.1180 & 1.0000 & 0.9487 & 1.1180 & 0.9487 \\
\hline 6 & 21 & 1.0000 & 1.0000 & 0.9220 & 1.0000 & 0.922 \\
\hline 11 & 21 & 1.0000 & 1.0000 & 0.7071 & 1.0000 & 0.7071 \\
\hline 2 & 22 & 1.4142 & 1.0911 & 0.9258 & 1.4142 & 0.9258 \\
\hline 4 & 22 & 1.2910 & 1.0911 & 0.9258 & 1.2910 & 0.9258 \\
\hline 8 & 22 & 1.0690 & 1.0911 & 0.8997 & 1.0690 & 0.8997 \\
\hline 2 & 23 & 1.4142 & 1.0871 & 0.9535 & 1.4142 & 0.9535 \\
\hline 3 & 23 & 1.0000 & 1.0871 & 0.9535 & 1.0000 & 0.9535 \\
\hline 12 & 23 & 1.0871 & 1.0871 & 0.8528 & 0.8528 & 1.0871 \\
\hline 2 & 24 & 1.4142 & 1.0632 & 1.0426 & 1.4142 & 1.042 \\
\hline 2 & 25 & 1.4142 & 1.0408 & 1.0408 & 1.4142 & 1.0408 \\
\hline 3 & 25 & 1.0000 & 1.0408 & 1.0000 & 1.0000 & 1.040 \\
\hline 4 & 25 & 1.2910 & 1.0408 & 1.0206 & 1.2910 & 1.0206 \\
\hline 5 & 25 & 1.1180 & 1.0408 & 0.9129 & 1.1180 & 0.9129 \\
\hline 7 & 25 & 1.1547 & 1.0408 & 0.8660 & 1.1547 & 0.8660 \\
\hline 9 & 25 & 1.1180 & 1.0408 & 1.0000 & 1.0000 & 1.0408 \\
\hline 13 & 25 & 1.0408 & 1.0408 & 1.0408 & 1.0408 & 1.040 \\
\hline 2 & 26 & 1.4142 & 1.0198 & 1.0198 & 1.4142 & 1.019 \\
\hline 6 & 26 & 1.0000 & 1.0198 & 1.0000 & 1.0000 & 1.000 \\
\hline 2 & 27 & 1.4142 & 1.0000 & 1.0000 & 1.4142 & 1.000 \\
\hline 3 & 27 & 1.0000 & 1.0000 & 1.0000 & 1.0000 & 1.000 \\
\hline 14 & 27 & 1.1435 & 1.0000 & 1.0000 & 1.0000 & 1.0000 \\
\hline 2 & 28 & 1.4142 & 1.0364 & 0.9813 & 1.4142 & 0.981 \\
\hline 4 & 28 & 1.2910 & 1.0364 & 0.9623 & 1.2910 & 0.962 \\
\hline 10 & 28 & 1.0541 & 1.0364 & 0.9428 & 0.9428 & 0.981 \\
\hline 2 & 29 & 1.4142 & 1.0177 & 0.9636 & 1.4142 & 0.963 \\
\hline 3 & 29 & 1.0000 & 1.0177 & 0.9636 & 1.0000 & 0.963 \\
\hline 5 & 29 & 1.1180 & 1.0177 & 0.9636 & 1.1180 & 0.963 \\
\hline 8 & 29 & 1.0690 & 1.0177 & 0.9449 & 1.0690 & 0.944 \\
\hline 15 & 29 & 1.1019 & 1.0177 & 0.9636 & 0.9636 & 0.963 \\
\hline 2 & 30 & 1.4142 & 1.0000 & 1.0000 & 1.4142 & 1.000 \\
\hline 2 & 31 & 1.4142 & 1.0328 & 0.9832 & 1.4142 & 0.983 \\
\hline 3 & 31 & 1.0000 & 1.0328 & 0.9832 & 1.0000 & 0.983 \\
\hline 4 & 31 & 1.2910 & 1.0328 & 0.9309 & 1.2910 & 0.930 \\
\hline 6 & 31 & 1.0000 & 1.0328 & 0.9309 & 1.0000 & 0.930 \\
\hline 7 & 31 & 1.1547 & 1.0328 & 0.9129 & 1.1547 & 0.912 \\
\hline 11 & 31 & 1.0000 & 1.0328 & 0.9309 & 1.0000 & 0.930 \\
\hline 16 & 31 & 1.0646 & 1.0328 & 0.9309 & 0.9309 & 0.9309 \\
\hline 2 & 32 & 1.4142 & 1.0160 & 0.9672 & 1.4142 & 0.967 \\
\hline
\end{tabular}


Table 10 Maximin distances for two-dimensional nested designs; $c_{2} \notin \mathbb{N}$

\begin{tabular}{|c|c|c|c|c|c|c|c|c|c|c|c|c|}
\hline \multirow[t]{2}{*}{$\overline{n_{1}}$} & \multirow[t]{2}{*}{$n_{2}$} & \multirow[t]{2}{*}{$d\left(n_{1}\right)$} & \multirow[t]{2}{*}{$d\left(n_{2}\right)$} & \multicolumn{3}{|c|}{ Nested $n_{1}$-grid } & \multicolumn{3}{|c|}{ Nested $n_{2}$-grid } & \multicolumn{3}{|c|}{ Grid with nested axes } \\
\hline & & & & $\bar{d}$ & $d_{1}$ & $d_{2}$ & $\bar{d}$ & $d_{1}$ & $d_{2}$ & $\bar{d}$ & $d_{1}$ & $d_{2}$ \\
\hline$\overline{3}$ & 4 & 1.0000 & 1.2910 & 0.6124 & 1.0000 & 0.6124 & 0.8165 & 1.3333 & 0.8165 & 0.6999 & 1.1429 & 0.6999 \\
\hline 4 & 5 & 1.2910 & 1.1180 & 1.0541 & 1.2910 & 1.0541 & 1.1180 & 1.3693 & 1.1180 & 1.0880 & 1.3325 & 1.0880 \\
\hline 3 & 6 & 1.0000 & 1.0000 & 0.9317 & 1.0000 & 0.9317 & 1.0000 & 1.2000 & 1.0000 & 0.9091 & 1.0909 & 0.9091 \\
\hline 4 & 6 & 1.2910 & 1.0000 & 0.8165 & 0.8165 & 1.0541 & 0.9798 & 0.9798 & 1.0000 & 0.8645 & 0.8645 & 1.1161 \\
\hline 5 & 6 & 1.1180 & 1.0000 & 0.8839 & 1.1180 & 0.8839 & 0.8944 & 0.8944 & 1.0000 & 0.9575 & 0.9722 & 0.9575 \\
\hline 5 & 7 & 1.1180 & 1.1547 & 0.9682 & 1.1180 & 0.9682 & 0.9428 & 0.9428 & 1.1547 & 0.9897 & 1.0302 & 0.9897 \\
\hline 6 & 7 & 1.0000 & 1.1547 & 1.0000 & 1.0000 & 1.0392 & 1.0541 & 1.0541 & 1.1547 & 1.1161 & 1.1161 & 1.1207 \\
\hline 3 & 8 & 1.0000 & 1.0690 & 0.9354 & 1.0000 & 0.9354 & 1.0690 & 1.1429 & 1.0690 & 0.9978 & 1.0667 & 0.9978 \\
\hline 4 & 8 & 1.2910 & 1.0690 & 0.7916 & 0.8165 & 0.7916 & 0.8452 & 1.3325 & 0.8452 & 0.7990 & 1.3152 & 0.7990 \\
\hline 5 & 8 & 1.1180 & 1.0690 & 1.0458 & 1.1180 & 1.0458 & 0.8452 & 1.0302 & 0.8452 & 0.9990 & 1.0968 & 0.9990 \\
\hline 6 & 8 & 1.0000 & 1.0690 & 0.8367 & 1.0000 & 0.8367 & 0.9035 & 0.9035 & 1.0690 & 0.9126 & 0.9383 & 0.9126 \\
\hline 7 & 8 & 1.1547 & 1.0690 & 0.9129 & 0.9129 & 0.9860 & 0.9897 & 0.9897 & 1.0690 & 1.0319 & 1.0319 & 1.0349 \\
\hline 4 & 9 & 1.2910 & 1.1180 & 0.8889 & 1.2910 & 0.8889 & 1.0000 & 1.2624 & 1.0000 & 0.9231 & 1.2814 & 0.9231 \\
\hline 6 & 9 & 1.0000 & 1.1180 & 0.8944 & 1.0000 & 0.8944 & 1.0000 & 1.0078 & 1.0000 & 0.9575 & 0.9575 & 0.9722 \\
\hline 7 & 9 & 1.1547 & 1.1180 & 0.9129 & 0.9129 & 1.0000 & 0.9682 & 0.9682 & 1.1180 & 0.9422 & 0.9422 & 1.0000 \\
\hline 8 & 9 & 1.0690 & 1.1180 & 0.8571 & 1.0690 & 0.8571 & 1.0458 & 1.0458 & 1.1180 & 0.9990 & 0.9990 & 1.0679 \\
\hline 3 & 10 & 1.0000 & 1.0541 & 0.8485 & 1.0000 & 0.8485 & 0.9428 & 1.1111 & 0.9428 & 0.8932 & 1.0526 & 0.8932 \\
\hline 5 & 10 & 1.1180 & 1.0541 & 0.7071 & 0.7071 & 0.8839 & 0.8012 & 0.8012 & 0.9428 & 0.7692 & 0.7692 & 0.9247 \\
\hline 6 & 10 & 1.0000 & 1.0541 & 0.9487 & 1.0000 & 0.9487 & 0.8958 & 0.8958 & 0.9428 & 0.9680 & 0.9798 & 0.9680 \\
\hline 7 & 10 & 1.1547 & 1.0541 & 0.7906 & 1.1547 & 0.7906 & 0.9428 & 0.9813 & 0.9428 & 0.8883 & 0.8883 & 0.9035 \\
\hline 8 & 10 & 1.0690 & 1.0541 & 0.8452 & 0.8452 & 0.9583 & 0.9296 & 0.9296 & 1.0541 & 0.9097 & 0.9226 & 0.9097 \\
\hline 9 & 10 & 1.1180 & 1.0541 & 0.9561 & 1.0000 & 0.9561 & 0.9938 & 0.9938 & 1.0541 & 0.9513 & 0.9513 & 1.0090 \\
\hline 4 & 11 & 1.2910 & 1.0000 & 0.8784 & 1.2910 & 0.8784 & 0.8944 & 1.1619 & 0.8944 & 0.8863 & 1.2103 & 0.8863 \\
\hline 5 & 11 & 1.1180 & 1.0000 & 0.7454 & 1.1180 & 0.7454 & 0.8944 & 1.0770 & 0.8944 & 0.8131 & 1.0985 & 0.8131 \\
\hline 7 & 11 & 1.1547 & 1.0000 & 0.8333 & 1.1547 & 0.8333 & 0.8944 & 1.0392 & 0.8944 & 0.8824 & 0.9666 & 0.8824 \\
\hline 8 & 11 & 1.0690 & 1.0000 & 0.8452 & 0.8452 & 1.0102 & 0.9539 & 0.9539 & 1.0000 & 0.8965 & 0.8965 & 1.0715 \\
\hline 9 & 11 & 1.1180 & 1.0000 & 1.0000 & 1.0000 & 1.0078 & 0.8944 & 1.0198 & 0.8944 & 0.9722 & 0.9722 & 1.0009 \\
\hline 10 & 11 & 1.0541 & 1.0000 & 0.9428 & 0.9428 & 0.9938 & 0.9487 & 0.9487 & 1.0000 & 0.9680 & 0.9680 & 0.9798 \\
\hline 3 & 12 & 1.0000 & 1.0871 & 0.8740 & 1.0000 & 0.8740 & 0.9535 & 1.0041 & 0.9535 & 0.9120 & 1.0009 & 0.9120 \\
\hline 4 & 12 & 1.2910 & 1.0871 & 0.9965 & 1.2910 & 0.9965 & 1.0871 & 1.2695 & 1.0871 & 1.0250 & 1.2838 & 1.0250 \\
\hline 5 & 12 & 1.1180 & 1.0871 & 0.7817 & 1.1180 & 0.7817 & 0.8528 & 1.0602 & 0.8528 & 0.7984 & 1.1039 & 0.7984 \\
\hline 6 & 12 & 1.0000 & 1.0871 & 0.9446 & 1.0000 & 0.9446 & 0.9535 & 1.0947 & 0.9535 & 0.9511 & 1.0699 & 0.9511 \\
\hline 7 & 12 & 1.1547 & 1.0871 & 0.8740 & 1.1547 & 0.8740 & 0.9535 & 0.9959 & 0.9535 & 0.8863 & 1.1222 & 0.8863 \\
\hline 8 & 12 & 1.0690 & 1.0871 & 0.8452 & 0.8452 & 1.0051 & 0.9535 & 1.0205 & 0.9535 & 0.8518 & 0.8518 & 1.0307 \\
\hline 9 & 12 & 1.1180 & 1.0871 & 1.0000 & 1.0000 & 1.0570 & 0.9271 & 0.9271 & 1.0871 & 0.9552 & 0.9552 & 0.9998 \\
\hline 10 & 12 & 1.0541 & 1.0871 & 0.9428 & 0.9428 & 1.0423 & 0.9833 & 0.9833 & 1.0871 & 0.9664 & 0.9664 & 0.9862 \\
\hline 11 & 12 & 1.0000 & 1.0871 & 1.0000 & 1.0000 & 1.0488 & 1.0365 & 1.0365 & 1.0871 & 1.0102 & 1.0102 & 1.0595 \\
\hline 6 & 13 & 1.0000 & 1.0408 & 0.9522 & 1.0000 & 0.9522 & 0.9129 & 1.0035 & 0.9129 & 0.9589 & 0.9589 & 0.9805 \\
\hline 8 & 13 & 1.0690 & 1.0408 & 0.8452 & 0.8452 & 1.0498 & 0.9129 & 0.9860 & 0.9129 & 0.8158 & 1.0380 & 0.8158 \\
\hline 9 & 13 & 1.1180 & 1.0408 & 0.9186 & 1.0000 & 0.9186 & 0.9129 & 1.0000 & 0.9129 & 0.8748 & 1.0000 & 0.8748 \\
\hline 10 & 13 & 1.0541 & 1.0408 & 0.9428 & 0.9428 & 0.9813 & 0.9129 & 1.0607 & 0.9129 & 0.9404 & 0.9583 & 0.9404 \\
\hline 11 & 13 & 1.0000 & 1.0408 & 0.8944 & 0.8944 & 0.9798 & 0.9501 & 0.9501 & 1.0408 & 0.9301 & 0.9301 & 0.9476 \\
\hline 12 & 13 & 1.0871 & 1.0408 & 0.9535 & 0.9535 & 0.9959 & 0.9965 & 0.9965 & 1.0408 & 0.9720 & 0.9720 & 1.0153 \\
\hline 3 & 14 & 1.0000 & 1.1435 & 0.9286 & 1.0000 & 0.9286 & 0.8771 & 1.0769 & 0.8771 & 0.9082 & 0.9630 & 0.9082 \\
\hline 4 & 14 & 1.2910 & 1.1435 & 0.9329 & 1.2910 & 0.9329 & 0.8771 & 1.3122 & 0.8771 & 0.8971 & 1.2280 & 0.8971 \\
\hline 5 & 14 & 1.1180 & 1.1435 & 0.8498 & 1.1180 & 0.8498 & 0.7845 & 1.1717 & 0.7845 & 0.8035 & 1.1557 & 0.8035 \\
\hline 6 & 14 & 1.0000 & 1.1435 & 0.8750 & 1.0000 & 0.8750 & 0.8771 & 1.0879 & 0.8771 & 0.8755 & 0.9722 & 0.8755 \\
\hline 7 & 14 & 1.1547 & 1.1435 & 0.9234 & 1.1547 & 0.9234 & 0.8771 & 1.0659 & 0.8771 & 0.8863 & 1.0851 & 0.8863 \\
\hline 8 & 14 & 1.0690 & 1.1435 & 0.8144 & 1.0690 & 0.8144 & 0.8771 & 1.0377 & 0.8771 & 0.8228 & 1.0801 & 0.8228 \\
\hline 9 & 14 & 1.1180 & 1.1435 & 0.7906 & 0.7906 & 1.0078 & 0.8971 & 0.8971 & 1.1435 & 0.8210 & 0.8210 & 1.0284 \\
\hline 10 & 14 & 1.0541 & 1.1435 & 0.9428 & 0.9428 & 1.0214 & 0.9515 & 0.9515 & 1.1435 & 0.9600 & 0.9600 & 1.0790 \\
\hline 11 & 14 & 1.0000 & 1.1435 & 0.9192 & 1.0000 & 0.9192 & 1.0030 & 1.0030 & 1.1435 & 0.9774 & 0.9774 & 1.1144 \\
\hline 12 & 14 & 1.0871 & 1.1435 & 0.9535 & 0.9535 & 1.0365 & 1.0519 & 1.0519 & 1.1435 & 1.0244 & 1.0244 & 1.0592 \\
\hline 13 & 14 & 1.0408 & 1.1435 & 1.0408 & 1.0408 & 1.0623 & 1.0987 & 1.0987 & 1.1435 & 1.0716 & 1.0716 & 1.1154 \\
\hline 4 & 15 & 1.2910 & 1.1019 & 0.8994 & 1.2910 & 0.8994 & 0.8748 & 0.8748 & 1.1019 & 0.9010 & 1.2852 & 0.9010 \\
\hline 5 & 15 & 1.1180 & 1.1019 & 0.8432 & 1.1180 & 0.8432 & 0.9636 & 1.1518 & 0.9636 & 0.8994 & 1.1333 & 0.8994 \\
\hline
\end{tabular}


Table 10 (continued)

\begin{tabular}{|c|c|c|c|c|c|c|c|c|c|c|c|c|}
\hline \multirow[t]{2}{*}{$\overline{n_{1}}$} & \multirow[t]{2}{*}{$n_{2}$} & \multirow[t]{2}{*}{$d\left(n_{1}\right)$} & \multirow[t]{2}{*}{$d\left(n_{2}\right)$} & \multicolumn{3}{|c|}{ Nested $n_{1}$-grid } & \multicolumn{3}{|c|}{ Nested $n_{2}$-grid } & \multicolumn{3}{|c|}{ Grid with nested axes } \\
\hline & & & & $d$ & $d_{1}$ & $d_{2}$ & $d$ & $d_{1}$ & $d_{2}$ & $\bar{d}$ & $d_{1}$ & $d_{2}$ \\
\hline 6 & 15 & 1.0000 & 1.1019 & 0.9080 & 1.0000 & 0.9080 & 0.8452 & 0.9313 & 0.8452 & 0.8960 & 0.9731 & 0.8960 \\
\hline 7 & 15 & 1.1547 & 1.1019 & 0.9582 & 1.1547 & 0.9582 & 1.1019 & 1.2372 & 1.1019 & 1.0456 & 1.2049 & 1.0456 \\
\hline 9 & 15 & 1.1180 & 1.1019 & 0.7906 & 0.7906 & 0.9922 & 0.8452 & 1.0880 & 0.8452 & 0.7967 & 0.7967 & 1.0242 \\
\hline 10 & 15 & 1.0541 & 1.1019 & 0.9428 & 0.9428 & 1.0599 & 0.9091 & 0.9091 & 0.9636 & 0.9299 & 0.9299 & 1.0758 \\
\hline 11 & 15 & 1.0000 & 1.1019 & 0.9539 & 1.0000 & 0.9539 & 0.9583 & 0.9583 & 0.9636 & 0.9272 & 0.9272 & 1.0295 \\
\hline 12 & 15 & 1.0871 & 1.1019 & 0.8672 & 1.0871 & 0.8672 & 0.9768 & 0.9768 & 1.1019 & 0.9675 & 0.9675 & 1.0147 \\
\hline 13 & 15 & 1.0408 & 1.1019 & 0.9129 & 0.9129 & 0.9860 & 1.0202 & 1.0202 & 1.1019 & 0.9923 & 0.9923 & 1.0718 \\
\hline 14 & 15 & 1.1435 & 1.1019 & 1.0000 & 1.0000 & 1.0176 & 1.0619 & 1.0619 & 1.1019 & 1.0368 & 1.0368 & 1.0759 \\
\hline
\end{tabular}

Table 11 Scaled separation distances for three-dimensional nested approximate maximin designs

\begin{tabular}{|c|c|c|c|c|c|c|c|c|c|c|c|c|}
\hline \multirow[t]{2}{*}{$n_{1}$} & \multirow[t]{2}{*}{$n_{2}$} & \multirow[t]{2}{*}{$d\left(n_{1}\right)$} & \multirow[t]{2}{*}{$d\left(n_{2}\right)$} & \multicolumn{3}{|c|}{ Nested $n_{1}$-grid } & \multicolumn{3}{|c|}{ Nested $n_{2}$-grid } & \multicolumn{3}{|c|}{ Grid with nested axes } \\
\hline & & & & $d$ & $d_{1}$ & $d_{2}$ & $d$ & $d_{1}$ & $d_{2}$ & $d$ & $d_{1}$ & $d_{2}$ \\
\hline$\overline{5}$ & 10 & 1.3162 & 1.2009 & 1.1400 & 1.1906 & 1.1400 & 1.0583 & 1.0583 & 1.0591 & 1.0990 & 1.0990 & 1.1664 \\
\hline 5 & 15 & 1.3162 & 1.1927 & 1.0827 & 1.3162 & 1.0827 & 1.1023 & 1.2524 & 1.1023 & 1.0661 & 1.2929 & 1.0661 \\
\hline 5 & 20 & 1.3162 & 1.1410 & 1.0506 & 1.1906 & 1.0506 & 1.0510 & 1.1991 & 1.0510 & 1.0888 & 1.3087 & 1.0888 \\
\hline 5 & 25 & 1.3162 & 1.1465 & 1.0546 & 1.1906 & 1.0546 & 1.0546 & 1.1906 & 1.0546 & 1.0546 & 1.1906 & 1.0546 \\
\hline 5 & 30 & 1.3162 & 1.1061 & 1.0582 & 1.1906 & 1.0582 & 1.0647 & 1.2708 & 1.0647 & 1.0672 & 1.1876 & 1.0672 \\
\hline 5 & 35 & 1.3162 & 1.1030 & 1.0605 & 1.1906 & 1.0605 & 1.0695 & 1.2148 & 1.0695 & 1.0530 & 1.1868 & 1.0530 \\
\hline 5 & 40 & 1.3162 & 1.1033 & 1.0623 & 1.1906 & 1.0623 & 1.0507 & 1.1811 & 1.0507 & 1.0587 & 1.1847 & 1.0587 \\
\hline 5 & 45 & 1.3162 & 1.0943 & 1.0553 & 1.1906 & 1.0553 & 1.0553 & 1.1906 & 1.0553 & 1.0553 & 1.1906 & 1.0553 \\
\hline 5 & 50 & 1.3162 & 1.0899 & 1.0517 & 1.1906 & 1.0517 & 1.0508 & 1.2095 & 1.0508 & 1.0537 & 1.1726 & 1.0537 \\
\hline 5 & 55 & 1.3162 & 1.0911 & 1.0510 & 1.1906 & 1.0510 & 1.0476 & 1.1857 & 1.0476 & 1.0471 & 1.1930 & 1.0471 \\
\hline 5 & 60 & 1.3162 & 1.0902 & 1.0431 & 1.1906 & 1.0431 & 1.0454 & 1.2020 & 1.0454 & 1.0492 & 1.3137 & 1.0492 \\
\hline 10 & 15 & 1.2009 & 1.1927 & 1.0285 & 1.0841 & 1.0285 & 1.0612 & 1.1119 & 1.0612 & 1.0396 & 1.0396 & 1.0559 \\
\hline 10 & 20 & 1.2009 & 1.1410 & 0.9895 & 1.0074 & 0.9895 & 1.0030 & 1.0034 & 1.0030 & 1.0114 & 1.0118 & 1.0114 \\
\hline 10 & 25 & 1.2009 & 1.1465 & 1.0036 & 1.0074 & 1.0036 & 1.0056 & 1.0218 & 1.0056 & 0.9895 & 0.9932 & 0.9895 \\
\hline 10 & 30 & 1.2009 & 1.1061 & 1.0074 & 1.0074 & 1.0118 & 1.0051 & 1.0566 & 1.0051 & 1.0052 & 1.0052 & 1.0092 \\
\hline 10 & 35 & 1.2009 & 1.1030 & 1.0221 & 1.0591 & 1.0221 & 1.0438 & 1.0684 & 1.0438 & 1.0182 & 1.0200 & 1.0182 \\
\hline 10 & 40 & 1.2009 & 1.1033 & 1.0074 & 1.0074 & 1.0231 & 1.0289 & 1.0411 & 1.0289 & 1.0272 & 1.0531 & 1.0272 \\
\hline 10 & 45 & 1.2009 & 1.0943 & 1.0108 & 1.0591 & 1.0108 & 1.0216 & 1.0216 & 1.0275 & 1.0224 & 1.0550 & 1.0224 \\
\hline 10 & 50 & 1.2009 & 1.0899 & 1.0201 & 1.0591 & 1.0201 & 1.0402 & 1.0748 & 1.0402 & 1.0183 & 1.0183 & 1.0230 \\
\hline 10 & 55 & 1.2009 & 1.0911 & 1.0119 & 1.0591 & 1.0119 & 1.0119 & 1.0591 & 1.0119 & 1.0119 & 1.0591 & 1.0119 \\
\hline 10 & 60 & 1.2009 & 1.0902 & 1.0129 & 1.0591 & 1.0129 & 1.0265 & 1.0303 & 1.0265 & 1.0345 & 1.0512 & 1.0345 \\
\hline 15 & 20 & 1.1927 & 1.1410 & 1.0612 & 1.0612 & 1.0908 & 1.0320 & 1.0383 & 1.0320 & 1.0431 & 1.0432 & 1.0431 \\
\hline 15 & 25 & 1.1927 & 1.1465 & 0.9889 & 0.9889 & 1.0250 & 0.9984 & 1.0092 & 0.9984 & 1.0146 & 1.0146 & 1.0255 \\
\hline 15 & 30 & 1.1927 & 1.1061 & 0.9889 & 0.9889 & 0.9996 & 0.9834 & 0.9834 & 0.9938 & 0.9824 & 0.9824 & 0.9994 \\
\hline 15 & 35 & 1.1927 & 1.1030 & 0.9889 & 0.9889 & 0.9945 & 0.9975 & 0.9975 & 0.9993 & 0.9822 & 0.9843 & 0.9822 \\
\hline 15 & 40 & 1.1927 & 1.1033 & 0.9889 & 0.9889 & 0.9889 & 0.9990 & 1.0117 & 0.9990 & 0.9942 & 0.9957 & 0.9942 \\
\hline 15 & 45 & 1.1927 & 1.0943 & 0.9889 & 0.9889 & 0.9981 & 0.9957 & 1.0130 & 0.9957 & 0.9914 & 0.9914 & 0.9970 \\
\hline 15 & 50 & 1.1927 & 1.0899 & 1.0038 & 1.0038 & 1.0041 & 0.9991 & 1.0140 & 0.9991 & 0.9990 & 1.0390 & 0.9990 \\
\hline 15 & 55 & 1.1927 & 1.0911 & 1.0034 & 1.0038 & 1.0034 & 1.0046 & 1.0275 & 1.0046 & 1.0176 & 1.0185 & 1.0176 \\
\hline 15 & 60 & 1.1927 & 1.0902 & 1.0283 & 1.0329 & 1.0283 & 1.0158 & 1.0221 & 1.0158 & 1.0111 & 1.0111 & 1.0128 \\
\hline 20 & 25 & 1.1410 & 1.1465 & 1.0600 & 1.0603 & 1.0600 & 1.0311 & 1.0311 & 1.0409 & 1.0240 & 1.0240 & 1.0290 \\
\hline 20 & 30 & 1.1410 & 1.1061 & 1.0224 & 1.0224 & 1.0322 & 1.0051 & 1.0080 & 1.0051 & 1.0097 & 1.0097 & 1.0135 \\
\hline 20 & 35 & 1.1410 & 1.1030 & 0.9758 & 1.0030 & 0.9758 & 0.9856 & 1.0051 & 0.9856 & 0.9858 & 0.9858 & 0.9868 \\
\hline 20 & 40 & 1.1410 & 1.1033 & 0.9570 & 0.9931 & 0.9570 & 0.9676 & 0.9676 & 0.9722 & 0.9711 & 0.9788 & 0.9711 \\
\hline 20 & 45 & 1.1410 & 1.0943 & 0.9831 & 0.9831 & 0.9915 & 0.9854 & 0.9854 & 0.9892 & 0.9750 & 0.9750 & 0.9750 \\
\hline 20 & 50 & 1.1410 & 1.0899 & 0.9909 & 0.9931 & 0.9909 & 0.9851 & 0.9938 & 0.9851 & 0.9854 & 0.9854 & 0.9872 \\
\hline 20 & 55 & 1.1410 & 1.0911 & 0.9908 & 1.0030 & 0.9908 & 0.9849 & 0.9895 & 0.9849 & 0.9888 & 0.9888 & 0.9924 \\
\hline 20 & 60 & 1.1410 & 1.0902 & 0.9831 & 0.9831 & 0.9897 & 0.9897 & 1.0011 & 0.9897 & 1.0013 & 1.0013 & 1.0117 \\
\hline
\end{tabular}


Table 11 (continued)

\begin{tabular}{|c|c|c|c|c|c|c|c|c|c|c|c|c|}
\hline \multirow[t]{2}{*}{$n_{1}$} & \multirow[t]{2}{*}{$n_{2}$} & \multirow[t]{2}{*}{$d\left(n_{1}\right)$} & \multirow[t]{2}{*}{$d\left(n_{2}\right)$} & \multicolumn{3}{|c|}{ Nested $n_{1}$-grid } & \multicolumn{3}{|c|}{ Nested $n_{2}$-grid } & \multicolumn{3}{|c|}{ Grid with nested axes } \\
\hline & & & & $d$ & $d_{1}$ & $d_{2}$ & $d$ & $d_{1}$ & $d_{2}$ & $\bar{d}$ & $d_{1}$ & $d_{2}$ \\
\hline$\overline{25}$ & 30 & 1.1465 & 1.1061 & 1.0546 & 1.0546 & 1.1068 & 1.0289 & 1.0289 & 1.0647 & 1.0367 & 1.0396 & 1.0367 \\
\hline 25 & 35 & 1.1465 & 1.1030 & 1.0339 & 1.0339 & 1.0521 & 1.0038 & 1.0038 & 1.0129 & 1.0081 & 1.0117 & 1.0081 \\
\hline 25 & 40 & 1.1465 & 1.1033 & 0.9984 & 0.9984 & 1.0066 & 0.9952 & 1.0033 & 0.9952 & 0.9980 & 0.9994 & 0.9980 \\
\hline 25 & 45 & 1.1465 & 1.0943 & 0.9618 & 0.9911 & 0.9618 & 0.9827 & 0.9834 & 0.9827 & 0.9877 & 0.9878 & 0.9877 \\
\hline 25 & 50 & 1.1465 & 1.0899 & 0.9491 & 0.9764 & 0.9491 & 0.9737 & 0.9797 & 0.9737 & 0.9778 & 0.9792 & 0.9778 \\
\hline 25 & 55 & 1.1465 & 1.0911 & 0.9744 & 0.9764 & 0.9744 & 0.9704 & 0.9704 & 0.9749 & 0.9725 & 0.9728 & 0.9725 \\
\hline 25 & 60 & 1.1465 & 1.0902 & 0.9764 & 0.9764 & 0.9796 & 0.9720 & 0.9827 & 0.9720 & 0.9671 & 0.9698 & 0.9671 \\
\hline 30 & 35 & 1.1061 & 1.1030 & 1.0647 & 1.0647 & 1.0759 & 1.0342 & 1.0342 & 1.0350 & 1.0267 & 1.0273 & 1.0267 \\
\hline 30 & 40 & 1.1061 & 1.1033 & 1.0271 & 1.0271 & 1.0508 & 1.0119 & 1.0119 & 1.0252 & 1.0043 & 1.0043 & 1.0247 \\
\hline 30 & 45 & 1.1061 & 1.0943 & 0.9995 & 0.9995 & 1.0094 & 0.9989 & 1.0022 & 0.9989 & 0.9897 & 0.9897 & 0.9930 \\
\hline 30 & 50 & 1.1061 & 1.0899 & 0.9825 & 0.9825 & 0.9936 & 0.9823 & 0.9894 & 0.9823 & 0.9810 & 0.9839 & 0.9810 \\
\hline 30 & 55 & 1.1061 & 1.0911 & 0.9357 & 0.9357 & 0.9466 & 0.9799 & 0.9805 & 0.9799 & 0.9823 & 0.9847 & 0.9823 \\
\hline 30 & 60 & 1.1061 & 1.0902 & 0.9113 & 0.9113 & 0.9179 & 0.9658 & 0.9658 & 0.9720 & 0.9773 & 0.9834 & 0.9773 \\
\hline 35 & 40 & 1.1030 & 1.1033 & 1.0653 & 1.0653 & 1.1151 & 1.0441 & 1.0441 & 1.0650 & 1.0322 & 1.0341 & 1.0322 \\
\hline 35 & 45 & 1.1030 & 1.0943 & 1.0306 & 1.0306 & 1.0525 & 1.0122 & 1.0122 & 1.0212 & 1.0062 & 1.0062 & 1.0113 \\
\hline 35 & 50 & 1.1030 & 1.0899 & 1.0129 & 1.0129 & 1.0139 & 1.0027 & 1.0027 & 1.0075 & 0.9980 & 0.9985 & 0.998 \\
\hline 35 & 55 & 1.1030 & 1.0911 & 0.9764 & 0.9764 & 0.9990 & 0.9840 & 0.9840 & 0.9849 & 0.9897 & 0.9897 & 0.9923 \\
\hline 35 & 60 & 1.1030 & 1.0902 & 0.9764 & 0.9764 & 0.9766 & 0.9838 & 0.9838 & 0.9919 & 0.9833 & 0.9833 & 0.9841 \\
\hline 40 & 45 & 1.1033 & 1.0943 & 1.0614 & 1.0614 & 1.1050 & 1.0483 & 1.0483 & 1.0553 & 1.0392 & 1.0392 & 1.0412 \\
\hline 40 & 50 & 1.1033 & 1.0899 & 1.0362 & 1.0362 & 1.0647 & 1.0242 & 1.0242 & 1.0267 & 1.0073 & 1.0087 & 1.0073 \\
\hline 40 & 55 & 1.1033 & 1.0911 & 1.0066 & 1.0066 & 1.0291 & 1.0046 & 1.0068 & 1.0046 & 0.9976 & 0.9976 & 1.0014 \\
\hline 40 & 60 & 1.1033 & 1.0902 & 0.9761 & 0.9761 & 0.9919 & 0.9897 & 0.9956 & 0.9897 & 0.9899 & 0.9899 & 0.9907 \\
\hline 45 & 50 & 1.0943 & 1.0899 & 1.0584 & 1.0584 & 1.0804 & 1.0416 & 1.0416 & 1.0508 & 1.0300 & 1.0300 & 1.0300 \\
\hline 45 & 55 & 1.0943 & 1.0911 & 1.0492 & 1.0492 & 1.0686 & 1.0119 & 1.0170 & 1.0119 & 1.0116 & 1.0116 & 1.0164 \\
\hline 45 & 60 & 1.0943 & 1.0902 & 1.0181 & 1.0181 & 1.0194 & 0.9977 & 0.9977 & 1.0007 & 0.9956 & 0.9983 & 0.9956 \\
\hline 50 & 55 & 1.0899 & 1.0911 & 1.0402 & 1.0402 & 1.0744 & 1.0277 & 1.0277 & 1.0499 & 1.0321 & 1.0330 & 1.0321 \\
\hline 50 & 60 & 1.0899 & 1.0902 & 1.0267 & 1.0267 & 1.0563 & 1.0172 & 1.0172 & 1.0265 & 1.0061 & 1.0068 & 1.0061 \\
\hline
\end{tabular}

Table 12 Scaled separation distances for four-dimensional nested approximate maximin designs

\begin{tabular}{|c|c|c|c|c|c|c|c|c|c|c|c|c|}
\hline \multirow[t]{2}{*}{$n_{1}$} & \multirow[t]{2}{*}{$n_{2}$} & \multirow[t]{2}{*}{$d\left(n_{1}\right)$} & \multirow[t]{2}{*}{$d\left(n_{2}\right)$} & \multicolumn{3}{|c|}{ Nested $n_{1}$-grid } & \multicolumn{3}{|c|}{ Nested $n_{2}$-grid } & \multicolumn{3}{|c|}{ Grid with nested axes } \\
\hline & & & & $d$ & $d_{1}$ & $d_{2}$ & $d$ & $d_{1}$ & $d_{2}$ & $\bar{d}$ & $d_{1}$ & $d_{2}$ \\
\hline$\overline{5}$ & 10 & 1.3693 & 1.3608 & 1.2141 & 1.2748 & 1.2141 & 1.2472 & 1.3240 & 1.2472 & 1.2201 & 1.3279 & 1.2201 \\
\hline 5 & 15 & 1.3693 & 1.3035 & 1.2069 & 1.2247 & 1.2069 & 1.2203 & 1.3325 & 1.2203 & 1.2001 & 1.3880 & 1.2001 \\
\hline 5 & 20 & 1.3693 & 1.2862 & 1.1683 & 1.2247 & 1.1683 & 1.1784 & 1.1839 & 1.1784 & 1.1888 & 1.2094 & 1.1888 \\
\hline 5 & 25 & 1.3693 & 1.2407 & 1.1738 & 1.3693 & 1.1738 & 1.1738 & 1.3693 & 1.1738 & 1.1738 & 1.3693 & 1.1738 \\
\hline 5 & 30 & 1.3693 & 1.2241 & 1.1689 & 1.3693 & 1.1689 & 1.1706 & 1.2250 & 1.1706 & 1.1612 & 1.2926 & 1.1612 \\
\hline 5 & 35 & 1.3693 & 1.2074 & 1.1735 & 1.2247 & 1.1735 & 1.1735 & 1.3323 & 1.1735 & 1.1588 & 1.2132 & 1.1588 \\
\hline 5 & 40 & 1.3693 & 1.1902 & 1.1558 & 1.2247 & 1.1558 & 1.1640 & 1.2146 & 1.1640 & 1.1577 & 1.2222 & 1.1577 \\
\hline 5 & 45 & 1.3693 & 1.1881 & 1.1560 & 1.2748 & 1.1560 & 1.1560 & 1.2748 & 1.1560 & 1.1560 & 1.2748 & 1.1560 \\
\hline 5 & 50 & 1.3693 & 1.1830 & 1.1459 & 1.2247 & 1.1459 & 1.1492 & 1.3715 & 1.1492 & 1.1428 & 1.3652 & 1.1428 \\
\hline 5 & 55 & 1.3693 & 1.1773 & 1.1490 & 1.2247 & 1.1490 & 1.1502 & 1.2642 & 1.1502 & 1.1475 & 1.2695 & 1.1475 \\
\hline 5 & 60 & 1.3693 & 1.1734 & 1.1463 & 1.3693 & 1.1463 & 1.1487 & 1.2699 & 1.1487 & 1.1378 & 1.2282 & 1.1378 \\
\hline 10 & 15 & 1.3608 & 1.3035 & 1.2347 & 1.2472 & 1.2347 & 1.1966 & 1.1995 & 1.1966 & 1.1871 & 1.1878 & 1.1871 \\
\hline 10 & 20 & 1.3608 & 1.2862 & 1.1599 & 1.1706 & 1.1599 & 1.1419 & 1.1710 & 1.1419 & 1.1265 & 1.1265 & 1.1327 \\
\hline 10 & 25 & 1.3608 & 1.2407 & 1.1564 & 1.1706 & 1.1564 & 1.1319 & 1.1319 & 1.1333 & 1.1365 & 1.1511 & 1.1365 \\
\hline 10 & 30 & 1.3608 & 1.2241 & 1.1410 & 1.1706 & 1.1410 & 1.1373 & 1.1473 & 1.1373 & 1.1362 & 1.1564 & 1.1362 \\
\hline 10 & 35 & 1.3608 & 1.2074 & 1.1482 & 1.1706 & 1.1482 & 1.1496 & 1.1672 & 1.1496 & 1.1362 & 1.1362 & 1.1374 \\
\hline 10 & 40 & 1.3608 & 1.1902 & 1.1359 & 1.1386 & 1.1359 & 1.1427 & 1.1700 & 1.1427 & 1.1389 & 1.1462 & 1.1389 \\
\hline 10 & 45 & 1.3608 & 1.1881 & 1.1364 & 1.1547 & 1.1364 & 1.1410 & 1.1497 & 1.1410 & 1.1371 & 1.1392 & 1.1371 \\
\hline
\end{tabular}


Table 12 (continued)

\begin{tabular}{|c|c|c|c|c|c|c|c|c|c|c|c|c|}
\hline \multirow[t]{2}{*}{$n_{1}$} & \multirow[t]{2}{*}{$n_{2}$} & \multirow[t]{2}{*}{$d\left(n_{1}\right)$} & \multirow[t]{2}{*}{$d\left(n_{2}\right)$} & \multicolumn{3}{|c|}{ Nested $n_{1}$-grid } & \multicolumn{3}{|c|}{ Nested $n_{2}$-grid } & \multicolumn{3}{|c|}{ Grid with nested axes } \\
\hline & & & & $d$ & $d_{1}$ & $d_{2}$ & $d$ & $d_{1}$ & $d_{2}$ & $d$ & $d_{1}$ & $d_{2}$ \\
\hline 10 & 50 & 1.3608 & 1.1830 & 1.1547 & 1.1547 & 1.1599 & 1.1454 & 1.1606 & 1.1454 & 1.1382 & 1.1425 & 1.1382 \\
\hline 10 & 55 & 1.3608 & 1.1773 & 1.1425 & 1.1547 & 1.1425 & 1.1425 & 1.1547 & 1.1425 & 1.1425 & 1.1547 & 1.1425 \\
\hline 10 & 60 & 1.3608 & 1.1734 & 1.1222 & 1.1222 & 1.1268 & 1.1391 & 1.1647 & 1.1391 & 1.1298 & 1.2066 & 1.1298 \\
\hline 15 & 20 & 1.3035 & 1.2862 & 1.2124 & 1.2124 & 1.2207 & 1.1741 & 1.1741 & 1.1886 & 1.1751 & 1.1751 & 1.1852 \\
\hline 15 & 25 & 1.3035 & 1.2407 & 1.1510 & 1.1560 & 1.1510 & 1.1341 & 1.1341 & 1.1407 & 1.1451 & 1.1456 & 1.1451 \\
\hline 15 & 30 & 1.3035 & 1.2241 & 1.1126 & 1.1139 & 1.1126 & 1.1059 & 1.1101 & 1.1059 & 1.1020 & 1.1150 & 1.1020 \\
\hline 15 & 35 & 1.3035 & 1.2074 & 1.1394 & 1.1394 & 1.1535 & 1.1139 & 1.1163 & 1.1139 & 1.1178 & 1.1178 & 1.1197 \\
\hline 15 & 40 & 1.3035 & 1.1902 & 1.1135 & 1.1225 & 1.1135 & 1.1117 & 1.1267 & 1.1117 & 1.1128 & 1.1128 & 1.1225 \\
\hline 15 & 45 & 1.3035 & 1.1881 & 1.1207 & 1.1225 & 1.1207 & 1.1060 & 1.1303 & 1.1060 & 1.1074 & 1.1074 & 1.1105 \\
\hline 15 & 50 & 1.3035 & 1.1830 & 1.1239 & 1.1309 & 1.1239 & 1.1184 & 1.1221 & 1.1184 & 1.1103 & 1.1157 & 1.1103 \\
\hline 15 & 55 & 1.3035 & 1.1773 & 1.1218 & 1.1309 & 1.1218 & 1.1112 & 1.1219 & 1.1112 & 1.1223 & 1.1234 & 1.1223 \\
\hline 15 & 60 & 1.3035 & 1.1734 & 1.1139 & 1.1139 & 1.1155 & 1.1156 & 1.1166 & 1.1156 & 1.1036 & 1.1036 & 1.1091 \\
\hline 20 & 25 & 1.2862 & 1.2407 & 1.1987 & 1.1987 & 1.2162 & 1.1671 & 1.1671 & 1.1702 & 1.1747 & 1.1747 & 1.1765 \\
\hline 20 & 30 & 1.2862 & 1.2241 & 1.1732 & 1.1732 & 1.1904 & 1.1401 & 1.1429 & 1.1401 & 1.1386 & 1.1386 & 1.1418 \\
\hline 20 & 35 & 1.2862 & 1.2074 & 1.1152 & 1.1313 & 1.1152 & 1.1155 & 1.1155 & 1.1162 & 1.1336 & 1.1336 & 1.1368 \\
\hline 20 & 40 & 1.2862 & 1.1902 & 1.0988 & 1.0988 & 1.1083 & 1.0912 & 1.0945 & 1.0912 & 1.0970 & 1.1097 & 1.0970 \\
\hline 20 & 45 & 1.2862 & 1.1881 & 1.1249 & 1.1260 & 1.1249 & 1.1013 & 1.1108 & 1.1013 & 1.0952 & 1.0952 & 1.1015 \\
\hline 20 & 50 & 1.2862 & 1.1830 & 1.1098 & 1.1098 & 1.1145 & 1.0971 & 1.0971 & 1.1000 & 1.0917 & 1.0917 & 1.0945 \\
\hline 20 & 55 & 1.2862 & 1.1773 & 1.1062 & 1.1098 & 1.1062 & 1.1010 & 1.1010 & 1.1078 & 1.0931 & 1.0931 & 1.0963 \\
\hline 20 & 60 & 1.2862 & 1.1734 & 1.0988 & 1.0988 & 1.1024 & 1.1123 & 1.1123 & 1.1156 & 1.0933 & 1.0933 & 1.0947 \\
\hline 25 & 30 & 1.2407 & 1.2241 & 1.2024 & 1.2024 & 1.2192 & 1.1600 & 1.1600 & 1.1651 & 1.1670 & 1.1670 & 1.1685 \\
\hline 25 & 35 & 1.2407 & 1.2074 & 1.1629 & 1.1629 & 1.1690 & 1.1297 & 1.1313 & 1.1297 & 1.1431 & 1.1431 & 1.1474 \\
\hline 25 & 40 & 1.2407 & 1.1902 & 1.1407 & 1.1407 & 1.1548 & 1.1227 & 1.1251 & 1.1227 & 1.1193 & 1.1193 & 1.1204 \\
\hline 25 & 45 & 1.2407 & 1.1881 & 1.0996 & 1.1143 & 1.0996 & 1.0951 & 1.0975 & 1.0951 & 1.1057 & 1.1057 & 1.1108 \\
\hline 25 & 50 & 1.2407 & 1.1830 & 1.0969 & 1.0990 & 1.0969 & 1.0946 & 1.0963 & 1.0946 & 1.0817 & 1.0817 & 1.0827 \\
\hline 25 & 55 & 1.2407 & 1.1773 & 1.1182 & 1.1182 & 1.1204 & 1.1021 & 1.1127 & 1.1021 & 1.0875 & 1.0898 & 1.0875 \\
\hline 25 & 60 & 1.2407 & 1.1734 & 1.1182 & 1.1182 & 1.1193 & 1.0916 & 1.0957 & 1.0916 & 1.0869 & 1.0869 & 1.0895 \\
\hline 30 & 35 & 1.2241 & 1.2074 & 1.1950 & 1.1950 & 1.1980 & 1.1522 & 1.1522 & 1.1605 & 1.1509 & 1.1513 & 1.1509 \\
\hline 30 & 40 & 1.2241 & 1.1902 & 1.1596 & 1.1596 & 1.1784 & 1.1258 & 1.1258 & 1.1264 & 1.1255 & 1.1266 & 1.1255 \\
\hline 30 & 45 & 1.2241 & 1.1881 & 1.1401 & 1.1401 & 1.1477 & 1.1076 & 1.1076 & 1.1091 & 1.1198 & 1.1198 & 1.1204 \\
\hline 30 & 50 & 1.2241 & 1.1830 & 1.1061 & 1.1146 & 1.1061 & 1.1105 & 1.1147 & 1.1105 & 1.1123 & 1.1123 & 1.1130 \\
\hline 30 & 55 & 1.2241 & 1.1773 & 1.0861 & 1.0972 & 1.0861 & 1.0987 & 1.0998 & 1.0987 & 1.0935 & 1.0956 & 1.0935 \\
\hline 30 & 60 & 1.2241 & 1.1734 & 1.0943 & 1.0943 & 1.1022 & 1.0822 & 1.0822 & 1.0835 & 1.0824 & 1.0824 & 1.0831 \\
\hline 35 & 40 & 1.2074 & 1.1902 & 1.1799 & 1.1799 & 1.2144 & 1.1567 & 1.1567 & 1.1622 & 1.1488 & 1.1493 & 1.1488 \\
\hline 35 & 45 & 1.2074 & 1.1881 & 1.1670 & 1.1670 & 1.1905 & 1.1365 & 1.1367 & 1.1365 & 1.1266 & 1.1266 & 1.1340 \\
\hline 35 & 50 & 1.2074 & 1.1830 & 1.1496 & 1.1496 & 1.1568 & 1.1184 & 1.1259 & 1.1184 & 1.1078 & 1.1078 & 1.1123 \\
\hline 35 & 55 & 1.2074 & 1.1773 & 1.1341 & 1.1341 & 1.1360 & 1.1062 & 1.1062 & 1.1067 & 1.1097 & 1.1097 & 1.1100 \\
\hline 35 & 60 & 1.2074 & 1.1734 & 1.1071 & 1.1071 & 1.1147 & 1.0952 & 1.0952 & 1.0976 & 1.0892 & 1.0892 & 1.0939 \\
\hline 40 & 45 & 1.1902 & 1.1881 & 1.1780 & 1.1780 & 1.2141 & 1.1556 & 1.1556 & 1.1574 & 1.1450 & 1.1457 & 1.1450 \\
\hline 40 & 50 & 1.1902 & 1.1830 & 1.1728 & 1.1728 & 1.1848 & 1.1243 & 1.1243 & 1.1262 & 1.1319 & 1.1329 & 1.1319 \\
\hline 40 & 55 & 1.1902 & 1.1773 & 1.1485 & 1.1498 & 1.1485 & 1.1146 & 1.1174 & 1.1146 & 1.1146 & 1.1146 & 1.1166 \\
\hline 40 & 60 & 1.1902 & 1.1734 & 1.1354 & 1.1354 & 1.1437 & 1.0986 & 1.0988 & 1.0986 & 1.1064 & 1.1064 & 1.1075 \\
\hline 45 & 50 & 1.1881 & 1.1830 & 1.1736 & 1.1736 & 1.2056 & 1.1407 & 1.1407 & 1.1454 & 1.1454 & 1.1454 & 1.1471 \\
\hline 45 & 55 & 1.1881 & 1.1773 & 1.1780 & 1.1780 & 1.2026 & 1.1225 & 1.1236 & 1.1225 & 1.1225 & 1.1225 & 1.1244 \\
\hline 45 & 60 & 1.1881 & 1.1734 & 1.1455 & 1.1455 & 1.1494 & 1.1121 & 1.1121 & 1.1126 & 1.1112 & 1.1112 & 1.1152 \\
\hline 50 & 55 & 1.1830 & 1.1773 & 1.1718 & 1.1718 & 1.1981 & 1.1449 & 1.1449 & 1.1480 & 1.1443 & 1.1443 & 1.1445 \\
\hline 50 & 60 & 1.1830 & 1.1734 & 1.1606 & 1.1606 & 1.2065 & 1.1254 & 1.1256 & 1.1254 & 1.1152 & 1.1152 & 1.1163 \\
\hline
\end{tabular}




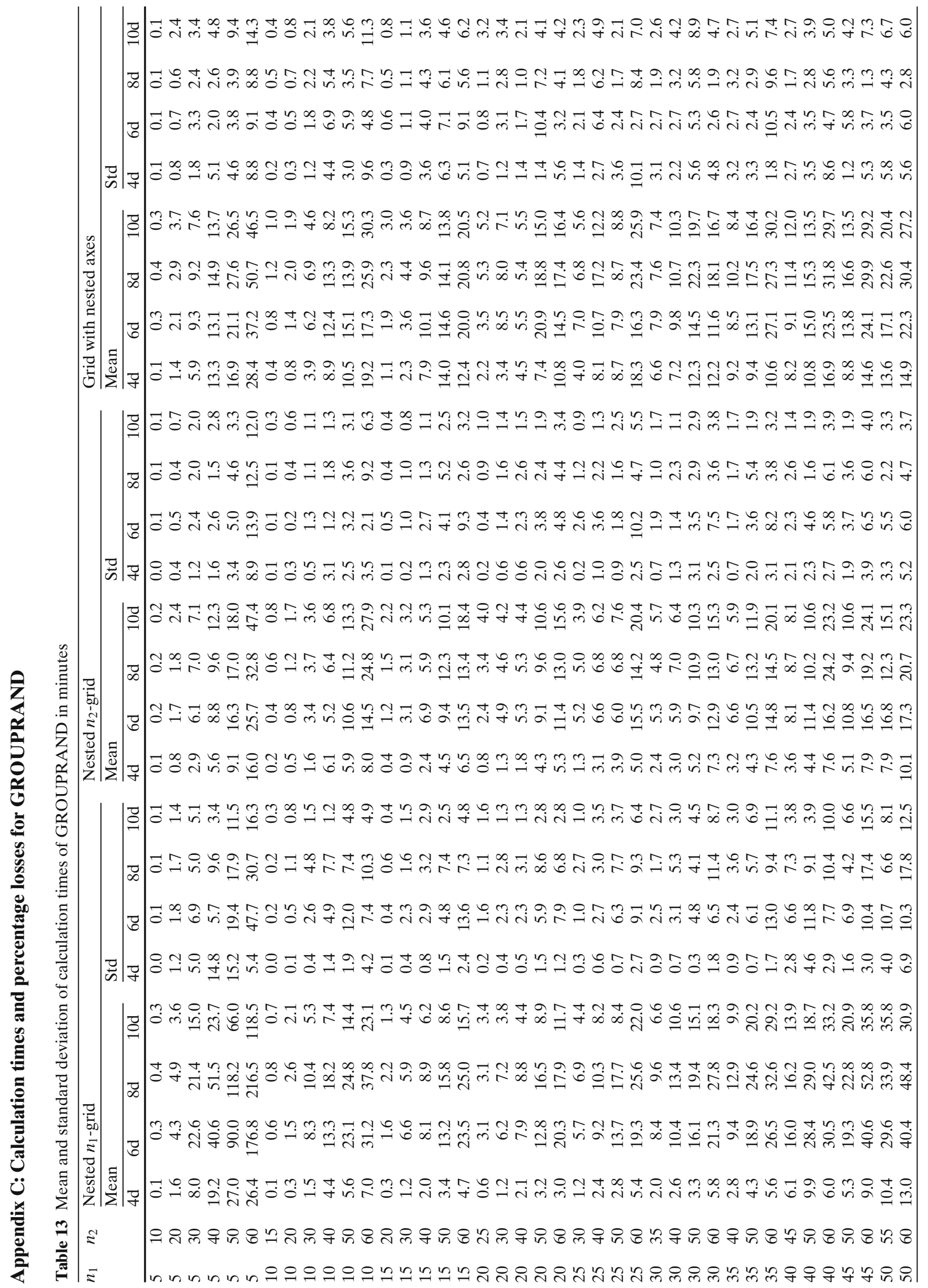




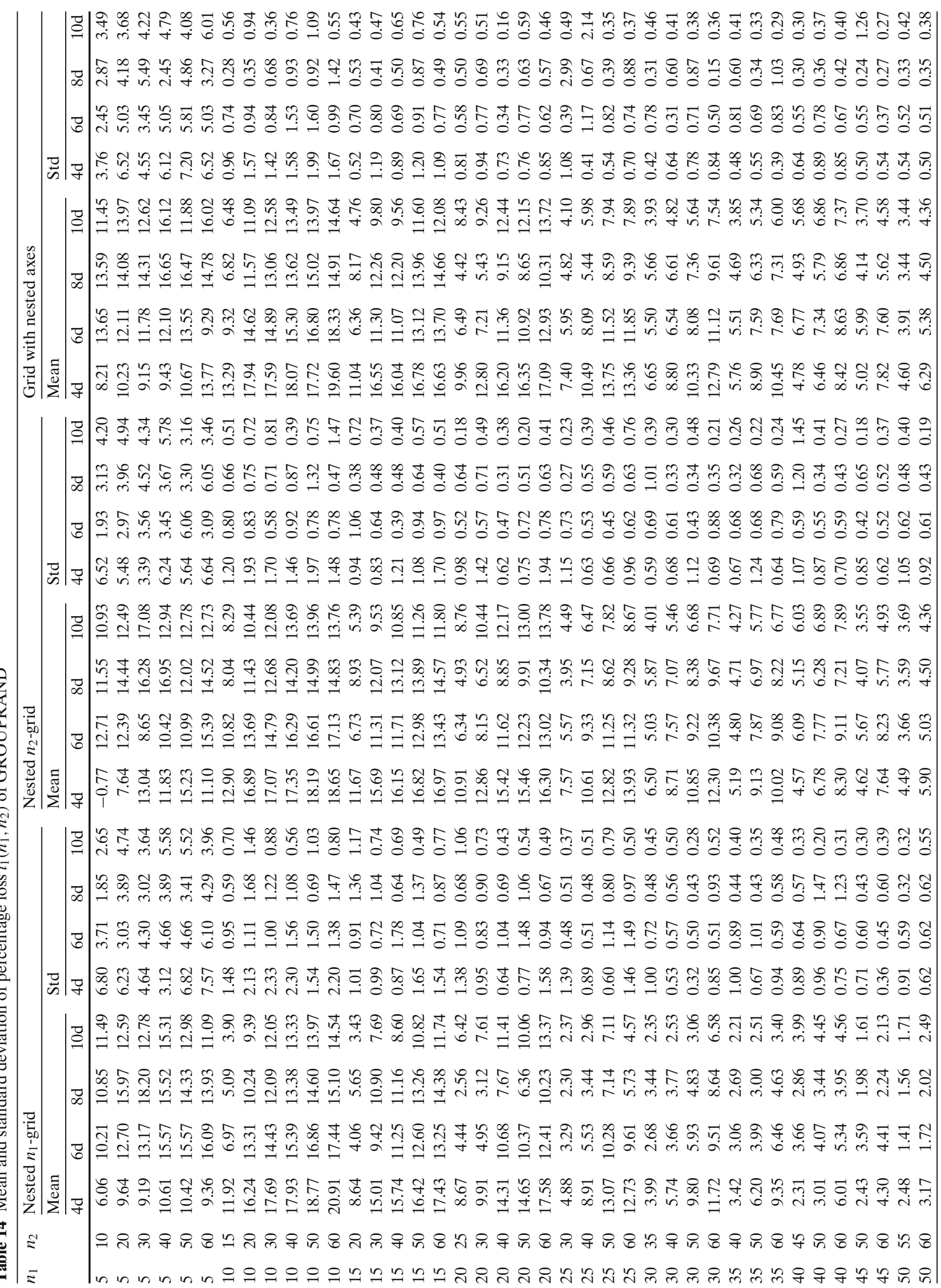




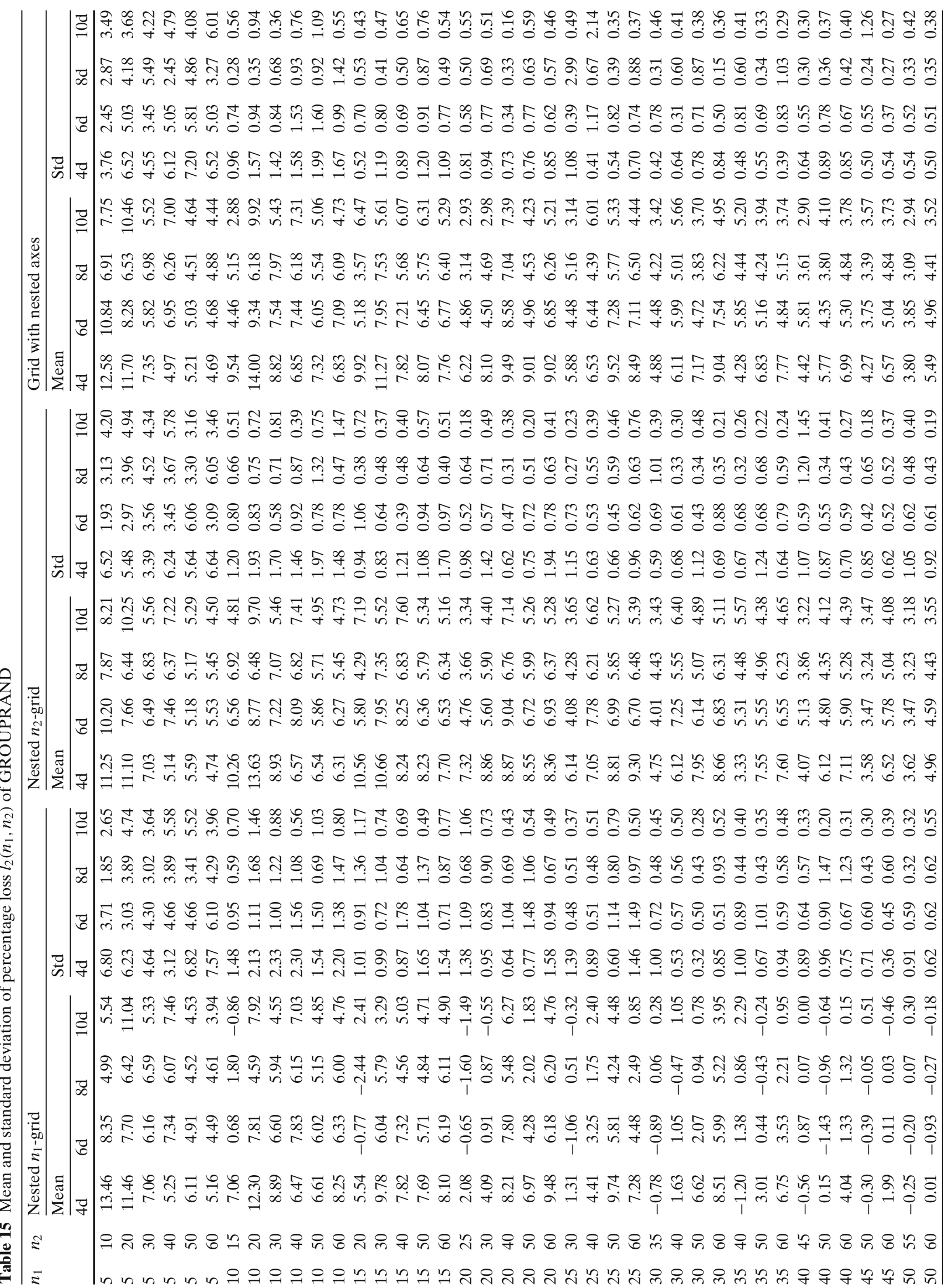




\section{References}

Barthelemy JFM, Haftka RT (1993) Approximation concepts for optimum structural design-a review. Struct Multidisc Optim 5(3):129-144

Booker AJ, Dennis JE, Frank PD, Serafini DB, Torczon V, Trosset MW (1999) A rigorous framework for optimization of expensive functions by surrogates. Struct Multidisc Optim 17(1):1-13

Cherkassky V, Mulier F (1998) Learning from data: concepts, theory, and methods. Wiley, New York

Cressie NAC (1993) Statistics for spatial data (revised ed), vol 605. Wiley, New York

Den Hertog D, Stehouwer HP (2002) Optimizing color picture tubes by high-cost nonlinear programming. Eur J Oper Res 140(2):197-211

Forrester AIJ, Keane AJ, Bressloff NW (2006) Design and analysis of "noisy" computer experiments. AIAA J 44(10): 2331-2339

Forrester AIJ, Sóbester A, Keane AJ (2007) Multi-fidelity optimization via surrogate modelling. In: Proceedings of the royal society a: mathematical, physical and engineering sciences, vol 463. The Royal Society, London, pp 3251-3269

Forrester AIJ, Sóbester A, Keane AJ (2008) Engineering design via surrogate modelling: a practical guide. Wiley, Chichester

Goel T, Haftka RT, Shyy W, Watson LT (2008) Pitfalls of using a single criterion for selecting experimental designs. Int $\mathbf{J}$ Numer Methods Eng 75(2):127-155

Grosso A, Jamali ARMJU, Locatelli M (2009) Finding maximin Latin hypercube designs by iterated local search heuristics. Eur J Oper Res 197(2):541-547

Husslage BGM, Van Dam ER, Den Hertog D, Stehouwer HP, Stinstra ED (2003) Collaborative metamodeling: coordinating simulation-based product design. Concurr Eng Res Appl 11(4):267-278

Husslage BGM, Van Dam ER, Den Hertog D (2005) Nested maximin Latin hypercube designs in two dimensions. CentER Discussion Paper 2005-79. Tilburg University, Tilburg, pp 1-11

Husslage BGM, Rennen G, Van Dam ER, Den Hertog D (2008) Space-filling Latin hypercube designs for computer experiments. CentER Discussion Paper 2008-104. Tilburg University, Tilburg, pp 1-14

Jin R, Chen W, Sudjianto A (2002) On sequential sampling for global metamodeling in engineering design. In: Proceedings of the ASME 2002 design engineering technical conferences and computers and information in engineering conference. Montreal, pp 1-10

Jin R, Chen W, Sudjianto A (2005) An efficient algorithm for constructing optimal design of computer experiments. J Stat Plan Inference 134(1):268-287

Johnson ME, Moore LM, Ylvisaker D (1990) Minimax and maximin distance designs. J Stat Plan Inference 26:131-148

Jones DR (2001) A taxonomy of global optimization methods based on response surfaces. J Glob Optim 21(4):345-383
Kennedy MC, O'Hagan A (2000) Predicting the output from a complex computer code when fast approximations are available. Biometrika 87(1):1-13

Kleijnen JPC (2008) Design and analysis of simulation experiments. In: International series in operations research \& management science, vol 111. Springer, New York

Montgomery DC (1984) Design and analysis of experiments, 2nd ed. Wiley, New York

Morris MD, Mitchell TJ (1995) Exploratory designs for computer experiments. J Stat Plan Inference 43:381-402

Myers RH (1999) Response surface methodology—current status and future directions. J Qual Technol 31:30-74

Qian Z, Seepersad CC, Joseph VR, Allen JK, Wu CFJ (2006) Building surrogate models based on detailed and approximate simulations. J Mech Des 128(4):668-677

Queipo NV, Haftka RT, Shyy W, Goel T, Vaidyanathan R, Tucker PK (2005) Surrogate-based analysis and optimization. Prog Aerosp Sci 41(1):1-28

Sacks J, Schiller SB, Welch WJ (1989a) Designs for computer experiments. Technometrics 31:41-47

Sacks J, Welch WJ, Mitchell TJ, Wynn HP (1989b) Design and analysis of computer experiments. Stat Sci 4:409-435

Santner ThJ, Williams BJ, Notz WI (2003) The design and analysis of computer experiments. Springer Series in Statistics. Springer, New York

Simpson TW, Booker AJ, Ghosh D, Giunta AA, Koch PN, Yang R-J (2004) Approximation methods in multidisciplinary analysis and optimization: a panel discussion. Struct Multidisc Optim 27(5):302-313

Simpson TW, Toropov VV, Balabanov V, Viana FAC (2008) Design and analysis of computer experiments in multidisciplinary design optimization: a review. In: Proceedings of the 12th AIAA/ISSMO multidisciplinary analysis and optimization conference, pp 1-22

Sobieszczanski-Sobieski J, Haftka RT (1997) Multidisciplinary aerospace design optimization: survey of recent developments. Struct Multidisc Optim 14(1):1-23

Van Dam ER, Husslage BGM, Den Hertog D, Melissen JBM (2007) Maximin Latin hypercube designs in two dimensions. Oper Res 55(1):158-169

Van Dam ER, Husslage BGM, Den Hertog D (2009a) Onedimensional nested maximin designs. J Glob Optim (in press)

Van Dam ER, Rennen G, Husslage BGM (2009b) Bounds for maximin Latin hypercube designs. Oper Res 57:595-608

Viana FAC, Balabanov V, Venter G, Garcelon J, Steffen V (2007) Generating optimal Latin hypercube designs in real time. In: 7th world congress on structural and multidisciplinary optimization, pp 2310-2315

Wang GG, Shan S (2007) Review of metamodeling techniques in support of engineering design optimization. $\mathrm{J}$ Mech Des 129(4):370-380

Ye KQ, Li W, Sudjianto A (2000) Algorithmic construction of optimal symmetric Latin hypercube designs. J Stat Plan Inference 90(1):145-159 